\title{
Homogeneity of the pure state space for separable $C^{*}$-algebras
}

\author{
Hajime Futamura, Nobuhiro Kataoka, and Akitaka Kishimoto \\ Department of Mathematics, Hokkaido University, Sapporo 060, Japan
}

January 2001

\begin{abstract}
We prove that the pure state space is homogeneous under the action of the automorphism group (or a certain smaller group of approximately inner automorphisms) for a fairly large class of simple separable nuclear $C^{*}$-algebras, including the approximately homogeneous $C^{*}$-algebras and the class of purely infinite $C^{*}$-algebras which has been recently classified by Kirchberg and Phillips. This extends the known results for UHF algebras and AF algebras by Powers and Bratteli.
\end{abstract}

Mathematics Subject Classification: 46L40, 46L30

\section{Introduction}

If $A$ is a $C^{*}$-algebra, we denote by $S(A)$ the convex set of states of $A$ and by $P(A)$ the set of pure states of $A$. The automorphism group $\operatorname{Aut}(A)$ of $A$ induces an action $\phi$ on $S(A)$ by affine homeomorphisms, i.e., if $\alpha \in \operatorname{Aut}(A)$, then $\phi(\alpha)$ sends $f \in S(A)$ to $f \alpha^{-1}$. Note that this action leaves $P(A)$ invariant since $P(A)$ is the set of extreme points of $S(A)$ and hence that it cannot be transitive on $S(A)$ (except for the trivial case $A=\mathrm{C} 1$ ). But from Powers' result [11] for UHF algebras we know that $\operatorname{Aut}(A)$ can act transitively on $P(A)$ for some simple $C^{*}$-algebras. See [1] for an immediate extension to $\mathrm{AF}$ algebras and [3] for a partial extension to Cuntz algebras.

Note that $\operatorname{Aut}(A)$ has some distinguished subgroups; $\operatorname{Inn}(A), \operatorname{AInn}(A), \overline{\operatorname{Inn}}(A)$. Denoting by $\mathcal{U}(A)$ the unitary group of $A$ (or $A+\mathbf{C} 1$ if $A \not \supset 1$ ), the group $\operatorname{Inn}(A)$ of inner automorphisms is given by $\{\operatorname{Ad} u \mid u \in \mathcal{U}(A)\}$ and the group $\overline{\operatorname{Inn}}(A)$ of approximately inner automorphisms is the closure of $\operatorname{Inn}(A)$ in $\operatorname{Aut}(A)$ with the topology of strong convergence. The group $\operatorname{AInn}(A)$ of asymptotically inner automorphisms consists of $\alpha \in \overline{\operatorname{Inn}}(A)$ such that there exists a continuous path $\left(u_{t}\right)_{t \in[0,1)}$ in $\mathcal{U}(A)$ with $\alpha=\lim _{t \rightarrow 1} \operatorname{Ad} u_{t}$; in the classification theory of purely infinite simple $C^{*}$-algebras [B], $\operatorname{AInn}(A)$ is characterized as the group of automorphisms which have the same KK class as the identity automorphism 
(see [9] for a similar characterization in the case of simple unital AT algebras of real rank zero). Note that $\operatorname{Inn}(A) \subset \operatorname{AInn}(A) \subset \overline{\operatorname{Inn}}(A) \subset \operatorname{Aut}(A)$ and that the inclusions are proper in general. In the statements given in the previous paragraph $\operatorname{Aut}(A)$ should be replaced by $\overline{\operatorname{Inn}}(A)$ from the way in which they are proven.

In this paper we aim to prove the following statement for a large class of separable $C^{*}$-algebras $A$ : If $\omega_{1}$ and $\omega_{2}$ are pure states of $A$ with ker $\pi_{\omega_{1}}=\operatorname{ker} \pi_{\omega_{2}}$, then there is an $\alpha \in \overline{\operatorname{Inn}}(A)$ such that $\omega_{1}=\omega_{2} \alpha$. Looking at the proofs closely, even more is true in the cases we will handle; it will follow that one can choose $\alpha$ from $\operatorname{AInn}(A)$. Hence, restricted to the case that the $C^{*}$-algebra $A$ is simple, we are to try to prove that $\operatorname{AInn}(A)$ acts on $P(A)$ transitively.

In the subsequent section we will introduce some properties for $C^{*}$-algebras and show that these properties imply the homogeneity of the pure state space in the above sense if the $C^{*}$-algebras are separable. This section contains the main idea of this paper.

In Sections 3-5 we will prove the above properties (more precisely, Property 2.9 below, the strongest among them) for a large class of $C^{*}$-algebras, namely approximately homogeneous $C^{*}$-algebras, simple crossed products of $\mathrm{AF}$ algebras by $\mathbf{Z}$, and a class of purely infinite $C^{*}$-algebras including the class which is classified by Kirchberg and Phillips [8, 10]. Hence the above-mentioned transitivity follows for these $C^{*}$-algebras. We might expect that this transitivity holds for all separable nuclear $C^{*}$-algebras. In section 6 we show that the transitivity holds also for the group $C^{*}$-algebras if the group is a countable discrete amenable group.

In Section 7 we note that even a stronger form of transitivity holds for the above mentioned separable $C^{*}$-algebras. One consequence is that given any sequence $\left(\pi_{n}\right)$, indexed by $\mathbf{Z}$, of distinct points in the set of equivalence classes of irreducible representations there is an asymptotically inner automorphism $\alpha$ which shifts this sequence, i.e., $\pi_{n} \alpha=\pi_{n+1}$ for all $n$.

The properties for $C^{*}$-algebras introduced in Section 2 seem interesting for their own sake. In Section 8 we will give yet another version, which is valid for the $C^{*}$-algebras treated above, to derive a closure property saying that if the property holds for a $C^{*}$ algebra, then it also holds for all its hereditary $C^{*}$-subalgebras. See 8.7 and 8.8 for other consequences.

The third named author would like to thank O. Bratteli for discussions at an early stage of this work.

\section{Homogeneity of the pure state space}

Let $A$ be a $C^{*}$-algebra and $\mathcal{U}(A)$ the unitary group of $A$ (or $A+\mathrm{C} 1$ if $A \not \nexists 1$ ). If two pure states $\omega_{1}$ and $\omega_{2}$ are equivalent or $\omega_{1} \sim \omega_{2}$, i.e., the GNS representation $\pi_{\omega_{1}}$ and $\pi_{\omega_{2}}$ are equivalent, then there is a $u \in \mathcal{U}(A)$ such that $\omega_{1}=\omega_{2} \operatorname{Ad} u$ by Kadison's transitivity (1.21.16 of [13]); we shall repeatedly use this fact below. First we define the following two properties for $A$ : 
Property 2.1 For any finite subset $\mathcal{F}$ of $A$ and $\epsilon>0$ there exist a finite subset $\mathcal{G}$ of $A$ and $\delta>0$ satisfying: If $\omega_{1}$ and $\omega_{2}$ are pure states of $A$ such that $\omega_{1} \sim \omega_{2}$ and

$$
\left|\omega_{1}(x)-\omega_{2}(x)\right|<\delta, \quad x \in \mathcal{G},
$$

then there is a $u \in \mathcal{U}(A)$ such that $\omega_{1}=\omega_{2} \operatorname{Ad} u$ and

$$
\|\operatorname{Ad} u(x)-x\|<\epsilon, \quad x \in \mathcal{F} .
$$

Property 2.2 For any finite subset $\mathcal{F}$ of $A$ and $\epsilon>0$ there exist a finite subset $\mathcal{G}$ of $A$ and $\delta>0$ satisfying: If $\omega_{1}$ and $\omega_{2}$ are pure states of $A$ such that $\operatorname{ker} \pi_{\omega_{1}}=\operatorname{ker} \pi_{\omega_{2}}$ and

$$
\left|\omega_{1}(x)-\omega_{2}(x)\right|<\delta, \quad x \in \mathcal{G},
$$

then for any finite subset $\mathcal{F}^{\prime}$ of $A$ and $\epsilon^{\prime}>0$ there is a $u \in \mathcal{U}(A)$ such that

$$
\begin{aligned}
\left|\omega_{1}(x)-\omega_{2} \operatorname{Ad} u(x)\right| & <\epsilon^{\prime}, \quad x \in \mathcal{F}^{\prime}, \\
\|\operatorname{Ad} u(x)-x\| & <\epsilon, \quad x \in \mathcal{F} .
\end{aligned}
$$

The following lemma is well-known (cf. [4]).

Lemma 2.3 Let $\omega_{1}$ and $\omega_{2}$ be pure states of $A$ such that $\operatorname{ker} \pi_{\omega_{1}}=\operatorname{ker} \pi_{\omega_{2}}$. For any finite subset $\mathcal{F}$ of $A$ and $\epsilon>0$ there is a $u \in \mathcal{U}(A)$ such that

$$
\left|\omega_{1}(x)-\omega_{2} \operatorname{Ad} u(x)\right|<\epsilon, \quad x \in \mathcal{F} .
$$

Proof. Since $\omega_{1}$ is a pure state, there exists an $e \in A$ such that $e \geq 0,\|e\|=1, \omega_{1}(e)=1$, and

$$
\left\|e x e-\omega_{1}(x) e^{2}\right\|<\epsilon, \quad x \in \mathcal{F} .
$$

Since $\left\|\pi_{\omega_{1}}(e)\right\|=1$, we may suppose, by slightly changing $e$ if necessary, that there is a $\xi \in \mathcal{H}_{\pi_{\omega_{2}}}$ such that $\|\xi\|=1$ and $\pi_{\omega_{2}}(e) \xi=\xi$. Since $\pi_{\omega_{2}}$ is irreducible there is a $u \in \mathcal{U}(A)$ such that $\pi_{\omega_{2}}\left(u^{*}\right) \Omega_{\omega_{2}}=\xi$. Since $\left\langle\pi_{\omega_{2}}(\right.$ exe $\left.) \xi, \xi\right\rangle=\omega_{2} \operatorname{Ad} u(x)$, we obtain the conclusion.

Proposition 2.4 For any $C^{*}$-algebra A Property 2.1 implies Property 2.9.

Proof. Suppose 2.1 and choose $(\mathcal{G}, \delta)$ for $(\mathcal{F}, \epsilon)$ as therein. Suppose that $\omega_{1}$ and $\omega_{2}$ are pure states of $A$ such that $\operatorname{ker} \pi_{\omega_{1}}=\operatorname{ker} \pi_{\omega_{2}}$ and

$$
\left|\omega_{1}(x)-\omega_{2}(x)\right|<\delta / 2, \quad x \in \mathcal{G} .
$$

Let $\mathcal{F}^{\prime}$ be a finite subset of $A$ and $\epsilon^{\prime}>0$ with $\epsilon^{\prime}<\delta / 2$. Then by Lemma 2.3 there is a $v \in \mathcal{U}(A)$ such that

$$
\left|\omega_{2} \operatorname{Ad} v(x)-\omega_{1}(x)\right|<\epsilon^{\prime}, \quad x \in \mathcal{F}^{\prime} \cup \mathcal{G} .
$$

Since $\left|\omega_{2} \operatorname{Ad} v(x)-\omega_{2}(x)\right|<\delta, x \in \mathcal{G}$, there is a $u \in \mathcal{U}(A)$ such that $\|\operatorname{Ad} u(x)-x\|<$ $\epsilon, x \in \mathcal{F}$ and $\omega_{2} \operatorname{Ad} v=\omega_{2} \operatorname{Ad} u$. The latter condition implies that $\left|\omega_{2} \operatorname{Ad} u(x)-\omega_{1}(x)\right|<$ $\epsilon^{\prime}, x \in \mathcal{F}^{\prime}$. This completes the proof. 
Theorem 2.5 Let $A$ be a separable $C^{*}$-algebra. Then the following conditions are equivalent:

1. Property 2.9 holds.

2. For any finite subset $\mathcal{F}$ of $A$ and $\epsilon>0$ there exist a finite subset $\mathcal{G}$ of $A$ and $\delta>0$ satisfying: If $\omega_{1}$ and $\omega_{2}$ are pure states of $A$ such that $\operatorname{ker} \pi_{\omega_{1}}=\operatorname{ker} \pi_{\omega_{2}}$ and $\left|\omega_{1}(x)-\omega_{2}(x)\right|<\delta, x \in \mathcal{G}$, there is an $\alpha \in \overline{\operatorname{Inn}}(A)$ such that $\omega_{1}=\omega_{2} \alpha$ and $\|\alpha(x)-x\|<\epsilon, x \in \mathcal{F}$. If $\mathcal{F}=\emptyset$, then $\mathcal{G}$ can be assumed to be empty.

Proof. First suppose $(2)$. For any $(\mathcal{F}, \epsilon)$ we choose $(\mathcal{G}, \delta)$ as in $(2)$. Let $\omega_{1}$ and $\omega_{2}$ be pure states of $A$ such that $\operatorname{ker} \pi_{\omega_{1}}=\operatorname{ker} \pi_{\omega_{2}}$ and $\left|\omega_{1}(x)-\omega_{2}(x)\right|<\delta, x \in \mathcal{G}$. Then there is an $\alpha \in \overline{\operatorname{Inn}}(A)$ as in (2). Since there is a sequence $\left(u_{n}\right)$ in $\mathcal{U}(A)$ such that $\alpha=\lim \operatorname{Ad} u_{n}$, it follows that for any finite subset $\mathcal{F}^{\prime}$ of $A$ and $\epsilon^{\prime}>0$ there is an $n$ such that $\left|\omega_{1}(x)-\omega_{2} \operatorname{Ad} u_{n}(x)\right|<\epsilon^{\prime}, x \in \mathcal{F}^{\prime}$ and $\left\|\operatorname{Ad} u_{n}(x)-x\right\|<\epsilon, x \in \mathcal{F}$. Thus (1) follows.

Suppose (1). Given $(\mathcal{F}, \epsilon)$ choose $(\mathcal{G}, \delta)$ as in Property 2.2 and let $\omega_{1}$ and $\omega_{2}$ be pure states of $A$ such that $\operatorname{ker} \pi_{\omega_{1}}=\operatorname{ker} \pi_{\omega_{2}}$ and $\left|\omega_{1}(x)-\omega_{2}(x)\right|<\delta, x \in \mathcal{G}$. (If $\mathcal{F}=\emptyset$, then we set $\mathcal{G}=\emptyset$, which will take care of the last statement.)

Let $\left(x_{n}\right)$ be a dense sequence in $A$.

Let $\mathcal{F}_{1}=\mathcal{F} \cup\left\{x_{1}\right\}$ and let $\left(\mathcal{G}_{1}, \delta_{1}\right)$ be the $(\mathcal{G}, \delta)$ for $\left(\mathcal{F}_{1}, \epsilon / 2\right)$ as in Property 2.2. By 2.2 there is a $u_{1} \in \mathcal{U}(A)$ such that

$$
\begin{aligned}
\left\|\operatorname{Ad} u_{1}(x)-x\right\| & <\epsilon, \quad x \in \mathcal{F}, \\
\left|\omega_{1}(x)-\omega_{2} \operatorname{Ad} u_{1}(x)\right| & <\delta_{1}, \quad x \in \mathcal{G}_{1} .
\end{aligned}
$$

Let $\mathcal{F}_{2}=\mathcal{F} \cup\left\{x_{1}, x_{2}, \operatorname{Ad} u_{1}^{*}\left(x_{1}\right), \operatorname{Ad} u_{1}^{*}\left(x_{2}\right)\right\}$ and let $\left(\mathcal{G}_{2}, \delta_{2}\right)$ be the $(\mathcal{G}, \delta)$ for $\left(\mathcal{F}_{2}, \epsilon / 2^{2}\right)$ as in Property 2.2 such that $\mathcal{G}_{2} \supset \mathcal{G}_{1}$ and $\delta_{2}<\delta_{1}$. Then there is a $u_{2} \in \mathcal{U}(A)$ such that

$$
\begin{aligned}
\left\|\operatorname{Ad} u_{2}(x)-x\right\| & <2^{-1} \epsilon, \quad x \in \mathcal{F}_{1}, \\
\left|\omega_{2} \operatorname{Ad} u_{1}(x)-\omega_{1} \operatorname{Ad} u_{2}(x)\right| & <\delta_{2}, \quad x \in \mathcal{G}_{2} .
\end{aligned}
$$

Let $\mathcal{F}_{3}=\mathcal{F} \cup\left\{x_{i}, \operatorname{Ad} u_{2}^{*}\left(x_{i}\right) \mid i=1,2,3\right\}$ and let $\left(\mathcal{G}_{3}, \delta_{3}\right)$ be the $(\mathcal{G}, \delta)$ for $\left(\mathcal{F}_{3}, 2^{-3} \epsilon\right)$ as in Property 2.2 such that $\mathcal{G}_{3} \supset \mathcal{G}_{2}$ and $\delta_{3}<\delta_{2}$. Then there is a $u_{3} \in \mathcal{U}(A)$ such that

$$
\begin{aligned}
\left\|\operatorname{Ad} u_{3}(x)-x\right\| & <2^{-2} \epsilon, \quad x \in \mathcal{F}_{2}, \\
\left|\omega_{1} \operatorname{Ad} u_{2}(x)-\omega_{2} \operatorname{Ad} u_{1} u_{3}(x)\right| & <\delta_{3}, \quad x \in \mathcal{G}_{3} .
\end{aligned}
$$

We shall repeat this process.

If $\mathcal{F}_{k}, \mathcal{G}_{k}, \delta_{k}$, and $u_{k}$ are given for $k<n$, let

$$
\mathcal{F}_{n}=\mathcal{F} \cup\left\{x_{i}, \operatorname{Ad} u_{n-1}^{*} u_{n-3}^{*} \cdots u_{\#}^{*}\left(x_{i}\right) \mid i=1,2, \ldots, n\right\},
$$

where $\#=2$ or 1 depending on the parity of $n$. We then choose $\left(\mathcal{G}_{n}, \delta_{n}\right)$ as in Property 2.2 for $\left(\mathcal{F}_{n}, 2^{-n} \epsilon\right)$ such that $\mathcal{G}_{n} \supset \mathcal{G}_{n-1}$ and $\delta_{n}<\delta_{n-1}$. Finally we pick up a $u_{n} \in \mathcal{U}(A)$ such that

$$
\left\|\operatorname{Ad} u_{n}(x)-x\right\|<2^{-n+1} \epsilon, \quad x \in \mathcal{F}_{n-1}
$$


and if $n$ is odd,

$$
\left|\omega_{1} \operatorname{Ad}\left(u_{2} u_{4} \cdots u_{n-1}\right)(x)-\omega_{2} \operatorname{Ad}\left(u_{1} u_{3} \cdots u_{n}\right)(x)\right|<\delta_{n}, \quad x \in \mathcal{G}_{n},
$$

else if $n$ is even,

$$
\left|\omega_{1} \operatorname{Ad}\left(u_{2} u_{4} \cdots u_{n}\right)(x)-\omega_{2} \operatorname{Ad}\left(u_{1} u_{3} \cdots u_{n-1}\right)(x)\right|<\delta_{n}, \quad x \in \mathcal{G}_{n}
$$

We may assume that $\cup_{n} \mathcal{G}_{n}$ is dense in $A$.

Since $\left\|\operatorname{Ad} u_{k}\left(x_{i}\right)-x_{i}\right\|<2^{-k+1} \epsilon$ for $k>i$, we have that

$$
\lim _{n \rightarrow \infty} \operatorname{Ad}\left(u_{1} u_{3} \cdots u_{2 n-1}\right)\left(x_{i}\right)
$$

converges for any $i$. Since $\left(x_{i}\right)$ is a dense sequence in $A$, we have that $\operatorname{Ad}\left(u_{1} u_{3} \cdots u_{2 n-1}\right)$ converges strongly on $A$; thus the limit $\alpha$ exists as an endomorphism of $A$. In a similar way $\operatorname{Ad}\left(u_{2} u_{4} \cdots u_{2 n}\right)$ converges to an endomorphism $\beta$ of $A$. From the estimate on $\omega_{1} \operatorname{Ad}\left(u_{2} u_{4} \cdots u_{2 n}\right)-\omega_{2} \operatorname{Ad}\left(u_{1} u_{3} \cdots u_{2 n+1}\right)$, it follows that $\omega_{1} \beta=\omega_{2} \alpha$.

We shall show that $\alpha$ and $\beta$ are automorphisms. For that purpose it suffices to show that $\operatorname{Ad}\left(u_{2 n}^{*} u_{2 n-2}^{*} \cdots u_{2}^{*}\right)$ and a similar expression with odd-numbered $u_{k}$ converge as $n \rightarrow \infty$.

Since $\left\|\operatorname{Ad} u_{n}(x)-x\right\|<2^{-n+1} \epsilon, x \in \mathcal{F}_{n-1}$, we have that if $n>i$,

$$
\left\|\operatorname{Ad} u_{n} \operatorname{Ad}\left(u_{n-2}^{*} u_{n-4}^{*} \cdots u_{\#}^{*}\right)\left(x_{i}\right)-\operatorname{Ad}\left(u_{n-2}^{*} u_{n-4}^{*} \cdots u_{\#}^{*}\right)\left(x_{i}\right)\right\|<2^{-n+1} \epsilon .
$$

This implies the desired convergence.

Finally, since $\mathcal{F}_{n} \supset \mathcal{F}$, it follows that the automorphisms $\alpha=\lim \operatorname{Ad}\left(u_{1} u_{3} \cdots u_{2 n-1}\right)$ and $\beta=\lim \operatorname{Ad}\left(u_{2} u_{4} \cdots u_{2 n}\right)$ satisfy that

$$
\|\alpha(x)-x\|<4 \epsilon / 3, \quad\|\beta(x)-x\|<2 \epsilon / 3,
$$

for $x \in \mathcal{F}$. Hence $\left\|\alpha \beta^{-1}(x)-x\right\|<2 \epsilon, x \in \mathcal{F}$. Since $\omega_{1}=\omega_{2} \alpha \beta^{-1}$, this completes the proof.

In the following sections we will actually treat properties stronger than 2.1.

Property 2.6 For any finite subset $\mathcal{F}$ of $A$ and $\epsilon>0$ there exist a finite subset $\mathcal{G}$ of $A$ and $\delta>0$ satisfying: If $\omega_{1}$ and $\omega_{2}$ are pure states of $A$ such that $\omega_{1} \sim \omega_{2}$ and

$$
\left|\omega_{1}(x)-\omega_{2}(x)\right|<\delta, \quad x \in \mathcal{G},
$$

then there is a continuous path $\left(u_{t}\right)_{t \in[0,1]}$ in $\mathcal{U}(A)$ such that $u_{0}=1, \omega_{1}=\omega_{2} \mathrm{Ad} u_{1}$, and

$$
\left\|\operatorname{Ad} u_{t}(x)-x\right\|<\epsilon, \quad x \in \mathcal{F}, t \in[0,1] .
$$

As in the proof of 2.4 we can show that the above property implies: 
Property 2.7 For any finite subset $\mathcal{F}$ of $A$ and $\epsilon>0$ there exist a finite subset $\mathcal{G}$ of $A$ and $\delta>0$ satisfying: If $\omega_{1}$ and $\omega_{2}$ are pure states of $A$ such that $\operatorname{ker} \pi_{\omega_{1}}=\operatorname{ker} \pi_{\omega_{2}}$ and

$$
\left|\omega_{1}(x)-\omega_{2}(x)\right|<\delta, \quad x \in \mathcal{G},
$$

then for any finite subset $\mathcal{F}^{\prime}$ of $A$ and $\epsilon^{\prime}>0$ there is a continuous path $\left(u_{t}\right)_{t \in[0,1]}$ in $\mathcal{U}(A)$ such that $u_{0}=1$, and

$$
\begin{aligned}
\left|\omega_{1}(x)-\omega_{2} \operatorname{Ad} u_{1}(x)\right| & <\epsilon^{\prime}, \quad x \in \mathcal{F}^{\prime}, \\
\left\|\operatorname{Ad} u_{t}(x)-x\right\| & <\epsilon, \quad x \in \mathcal{F}, t \in[0,1] .
\end{aligned}
$$

We recall here that $\operatorname{AInn}(A)$ is the group of asymptotically inner automorphisms of $A$, a proper normal subgroup of $\overline{\operatorname{Inn}}(A)$ in general. The following result can be shown exactly in the same way as Theorem 2.5 is shown:

Theorem 2.8 Let $A$ be a separable $C^{*}$-algebra. Then the following conditions are equivalent:

1. Property 2.7 holds.

2. For any finite subset $\mathcal{F}$ of $A$ and $\epsilon>0$ there exist a finite subset $\mathcal{G}$ of $A$ and $\delta>0$ satisfying: If $\omega_{1}$ and $\omega_{2}$ are pure states of $A$ such that $\operatorname{ker} \pi_{\omega_{1}}=\operatorname{ker} \pi_{\omega_{2}}$ and $\left|\omega_{1}(x)-\omega_{2}(x)\right|<\delta, x \in \mathcal{G}$, there exist an $\alpha \in \operatorname{AInn}(A)$ and a continuous path $\left(u_{t}\right)_{t \in[0,1)}$ in $\mathcal{U}(A)$ such that $u_{0}=1, \alpha=\lim _{t \rightarrow 1} \operatorname{Ad} u_{t}, \omega_{1}=\omega_{2} \alpha$ and $\| \operatorname{Ad} u_{t}(x)-$ $x \|<\epsilon, x \in \mathcal{F}, t \in[0,1)$. If $\mathcal{F}=\emptyset$, then $\mathcal{G}$ can be assumed to be empty.

Proof. The proof proceeds exactly in the same way as the proof of 2.5. In the proof of $(1) \Rightarrow(2)$ of 2.5 we have defined the automorphisms $\alpha$ and $\beta$ of $A$; the $\alpha$ in the above statement is $\alpha \beta^{-1}$. The $\alpha$ in the proof of 2.5 is defined as the limit of $\operatorname{Ad}\left(u_{1} u_{3} \cdots u_{2 n-1}\right)$. In the present assumption we have a continuous path $\left(u_{n t}\right)$ in $\mathcal{U}(A)$ for each $n$ such that $u_{n 0}=1, u_{n 1}=u_{n}$, and $\left\|\operatorname{Ad} u_{n t}(x)-x\right\|<2^{-n+1} \epsilon, x \in \mathcal{F}_{n-1}$. We define a continuous path $\left(v_{t}\right)_{t \in[0, \infty)}$ by: for $t \in[n, n+1]$,

$$
v_{t}=u_{1} u_{3} \cdots u_{2 n-1} u_{2 n+1, t-n} .
$$

Then it follows that $v_{0}=1, \alpha=\lim _{t \rightarrow \infty} \operatorname{Ad} v_{t}$, and $\left\|\operatorname{Ad} v_{t}(x)-x\right\| \leq 4 \epsilon / 3, x \in \mathcal{F}$. We prove that $\beta$ also enjoys a similar property and thus $\alpha \beta^{-1}$ does too. This shows that $(1) \Rightarrow(2)$.

The other implication is obvious since it is assumed that $u_{0}=1$ for the path $\left(u_{t}\right)$ in (2).

We will consider even a stronger property: 
Property 2.9 For any finite subset $\mathcal{F}$ of $A$ and $\epsilon>0$ there is a finite subset $\mathcal{G}$ of $A$ and $\delta>0$ satisfying: If $B$ is a $C^{*}$-algebra containing $A$ as a $C^{*}$-subalgebra and $\omega_{1}$ and $\omega_{2}$ are pure states of $B$ such that $\omega_{1} \sim \omega_{2}$ and

$$
\left|\omega_{1}(x)-\omega_{2}(x)\right|<\delta, \quad x \in \mathcal{G},
$$

then there is a continuous path $\left(u_{t}\right)_{t \in[0,1]}$ in $\mathcal{U}(B)$ such that $u_{0}=1, \omega_{1}=\omega_{2} \operatorname{Ad} u_{1}$, and

$$
\left\|\operatorname{Ad} u_{t}(x)-x\right\|<\epsilon, \quad x \in \mathcal{F}, t \in[0,1] .
$$

In the above property we may replace the exact equality $\omega_{1}=\omega_{2} \mathrm{Ad} u_{1}$ by an approximate one $\left\|\omega_{1}-\omega_{2} \operatorname{Ad} u_{1}\right\|<\epsilon$; by Kadison's transitivity one can make a small modification to $\left(u_{t}\right)$ to get the exact equality.

Obviously $2.9 \Leftrightarrow 2.6 \Leftrightarrow 2.7$ among the properties defined above.

Lemma 2.10 When $A$ is unital, Property 2.9 is equivalent to the one obtained by restricting the ambient algebra $B$ to a $C^{*}$-algebra having $1_{A}$ as a unit.

Proof. Given $(\mathcal{F}, \epsilon)$ let $(\mathcal{G}, \delta)$ be the one obtained in this weaker property. Let $\mathcal{G}_{1}=$ $\mathcal{G} \cup\left\{N 1_{A}\right\}$, where $N$ will be specified later to be a large positive number, and suppose that $B$ is given such that $B \supset A$ and $1_{A}$ is not an identity for $B$. If $\pi$ is an irreducible representation of $B$ and $\omega_{1}, \omega_{2}$ are pure states of $B$ defined by the unit vectors $\xi_{1}, \xi_{2} \in \mathcal{H}_{\pi}$ respectively such that $\left|\omega_{1}(x)-\omega_{2}(x)\right|<2^{-1} \epsilon^{2} \delta, x \in \mathcal{G}_{1}$, then we can choose $N$ so large that $\left|\omega_{1}\left(1_{A}\right)-\omega_{2}\left(1_{A}\right)\right|$ is very small and either, $\omega_{1}\left(1_{A}\right)$ and $\omega_{2}\left(1_{A}\right)$ are smaller than $\epsilon^{2}$, or $\left|\phi_{1}(x)-\phi_{2}(x)\right|<\delta, x \in \mathcal{G}$ for the states $\phi_{i}=\omega_{i}\left(1_{A}\right)^{-1} \omega_{i} \mid 1_{A} B 1_{A}$. In any case we can choose a continuous path $\left(w_{t}\right)_{t \in[0,1]}$ in $\mathcal{U}\left(\left(1-1_{A}\right) B\left(1-1_{A}\right)\right)$ such that $\left\|\left(1-\pi\left(1_{A}\right)\right) \xi_{1}-\pi\left(w_{1}\right) \xi_{2}\right\|$ is very small. In the former case where $\left\|\pi\left(1_{A}\right) \xi_{i}\right\|<\epsilon$, we set $v_{t}=1_{A}$ and in the latter case we choose, by the weaker property, a continuous path $\left(v_{t}\right)_{t \in[0,1]}$ in $\mathcal{U}\left(1_{A} B 1_{A}\right)$ such that $\left\|\pi\left(1_{A}\right) \xi_{1}-\pi\left(v_{1}\right) \xi_{2}\right\|$ is very small and $\left\|\operatorname{Ad} v_{t}(x)-x\right\|<\epsilon, x \in \mathcal{F}$. Setting $u_{t}=w_{t}+v_{t}$, it follows that $\left\|\xi_{1}-\pi\left(u_{1}\right) \xi_{2}\right\|$ is of order $\epsilon$ and $\left\|\operatorname{Ad} u_{t}(x)-x\right\|<\epsilon, x \in \mathcal{F}$. This completes the proof.

Proposition 2.11 If $\mathcal{C}$ denotes the class of $C^{*}$-algebras with Property 2.9, then the following statements hold:

1. If $A \in \mathcal{C}$ is non-unital, then $\tilde{A} \in \mathcal{C}$, where $\tilde{A}$ is the $C^{*}$-algebra obtained by adjoining a unit to $A$.

2. If $A_{1}, A_{2} \in \mathcal{C}$ are unital, then $A_{1} \oplus A_{2} \in \mathcal{C}$.

3. If $A \in \mathcal{C}$ and $e \in A$ is a projection, then $e A e \in \mathcal{C}$.

4. If $A \in \mathcal{C}$ and $I$ is an ideal of $A$, then the quotient $A / I \in \mathcal{C}$.

5. If $\left(A_{n}\right)$ is an inductive system with $A_{n} \in \mathcal{C}$, then $\lim _{n} A_{n} \in \mathcal{C}$. 
Proof. To prove (1) we may assume that $1_{B}=1_{\tilde{A}}$ in Property 2.9. Then this is obvious.

To prove (2) let $\mathcal{F}$ be a finite subset of $A \equiv A_{1} \oplus A_{2}$ and $\epsilon>0$. Then there is a finite subset $\mathcal{F}_{i} \subset A_{i}$ for each $i=1,2$ such that if $\|\operatorname{Ad} u(x)-x\|<\epsilon, x \in \mathcal{F}_{1} \cup \mathcal{F}_{2}$, then $\|\operatorname{Ad} u(x)-x\|<\epsilon, x \in \mathcal{F}$, for any $u \in \mathcal{U}(B)$ with $B \supset A$ and $1_{B}=1_{A}$. Choose $\left(\mathcal{G}_{i}, \delta_{i}\right)$ for $\left(\mathcal{F}_{i}, \epsilon\right)$ as in Property 2.9. We may suppose that $\delta_{1}=\delta_{2} \equiv \delta$ by multiplying each element of $\mathcal{G}_{1}$ by $\delta_{2} / \delta_{1}$. Let $\mathcal{G}=\mathcal{G}_{1} \cup \mathcal{G}_{2} \cup\left\{N p_{1}, N p_{2}\right\}$, where $p_{i}$ is the identity of $A_{i}$ in $B$ (and so $\left.p_{1}+p_{2}=1\right)$. Let $\pi$ be an irreducible representation of $B$ and let $\omega_{i}$ be a pure state of $B$ defined by a unit vector $\xi_{i} \in \mathcal{H}_{\pi}$ for $i=1,2$.

By choosing $N$ sufficiently large, we may assume that if $\left|\omega_{1}(x)-\omega_{2}(x)\right|<\epsilon^{2} \delta / 2, x \in \mathcal{G}$, then it follows that $\left|\omega_{1}\left(p_{i}\right)-\omega_{2}\left(p_{i}\right)\right|$ is very small for $i=1,2$, and both $\omega_{1}\left(p_{i}\right)$ and $\omega_{2}\left(p_{i}\right)$ are smaller than $\epsilon^{2}$ or

$$
\left|\frac{\omega_{1}(x)}{\omega_{1}\left(p_{i}\right)}-\frac{\omega_{2}(x)}{\omega_{2}\left(p_{i}\right)}\right|<\delta, \quad x \in \mathcal{G}_{i} .
$$

In the former case we set $u_{i t}=p_{i}$ and in the latter case we obtain a continuous path $\left(u_{i t}\right)$ in $\mathcal{U}\left(p_{i} B p_{i}\right)$ such that $u_{i 0}=p_{i}$, and

$$
\begin{aligned}
\left\|\operatorname{Ad} u_{i t}(x)-x\right\| & <\epsilon, \quad x \in \mathcal{F}_{i} \\
\omega_{1}\left(p_{i}\right)^{-1} \omega_{1} \mid p_{i} B p_{i} & =\omega_{2}\left(p_{i}\right)^{-1} \omega_{2} \operatorname{Ad} u_{i 1} \mid p_{i} B p_{i} .
\end{aligned}
$$

By multiplying $\left(u_{i t}\right)$ by a $\mathbf{T}$-valued continuous function, we may further suppose that $\pi\left(u_{i 1}\right) \xi_{1}=\left\|\pi\left(p_{i}\right) \xi_{1}\right\|\left\|\pi\left(p_{i}\right) \xi_{2}\right\|^{-1} \pi\left(p_{i}\right) \xi_{2}$, where $\left\|\pi\left(p_{i}\right) \xi_{1}\right\|\left\|\pi\left(p_{i}\right) \xi_{2}\right\|^{-1}$ is arbitrarily close to 1. Set $u_{t}=u_{1 t}+u_{2 t}$; then $\left\|\omega_{1}-\omega_{2} \operatorname{Ad} u_{1}\right\|$ is of the order of $\epsilon$ (since $\left\|\pi\left(u_{1}\right) \xi_{1}-\xi_{2}\right\|$ is at most of order $\epsilon$ ) and $\left\|\operatorname{Ad} u_{t}(x)-x\right\|<\epsilon, x \in \mathcal{F}_{1} \cup \mathcal{F}_{2}$.

To prove (3) let $e$ be a projection in $A \in \mathcal{C}$ and let $B$ be a $C^{*}$-algebra with $B \supset e A e$ and $1_{B}=e$. Then there is a $C^{*}$-algebra $D$ such that $e D e=B$ and the diagram

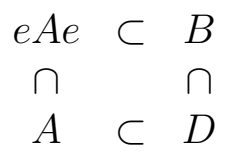

is commutative. (To show this we may suppose that $A$ acts on a Hilbert space $\mathcal{H}$ and that $B$ acts on $e \mathcal{H}$ with $B \supset e A e$. We set $D$ to be the $C^{*}$-algebra generated by $B$ and $A$.) Let $\mathcal{F}$ be a finite subset of $e A e$ and $\epsilon>0$. Let $\mathcal{F}_{1}=\mathcal{F} \cup\{N e\}$ with $N$ sufficiently large. We choose $(\mathcal{G}, \delta)$ for $\left(\mathcal{F}_{1}, \epsilon\right)$ from Property 2.9 of $A$ and set $\mathcal{G}_{1}=\{$ exe $\mid x \in \mathcal{G}\}$. Let $\omega_{1}, \omega_{2}$ be pure states of $B$ such that $\omega_{1} \sim \omega_{2}$ and $\left|\omega_{1}(x)-\omega_{2}(x)\right|<\delta, x \in \mathcal{G}_{1}$. Extend $\omega_{i}$ to a pure state of $D$; since the extension is unique, we denote it by the same symbol. Since $\omega_{i}(x)=\omega_{i}($ exe $)$, we obtain, from Property 2.9, a continuous path $\left(u_{t}\right)$ in $\mathcal{U}(D)$ such that $u_{0}=1, \omega_{1}=\omega_{2} \operatorname{Ad} u_{1}$, and $\left\|\operatorname{Ad} u_{t}(x)-x\right\|<\epsilon, x \in \mathcal{F}_{1}$. Since $\left\|\left[e, u_{t}\right]\right\|$ is very small, we can obtain a continuous path in $\mathcal{U}(e A e)$ from $\left(e u_{t} e\right)$ which satisfies the required properties.

To prove (4) let $B$ a $C^{*}$-algebra with $B \supset A / I$. Let $D=B \oplus A$ and embed $A$ into $D$ by $x \mapsto(x+I, x)$. Let $\mathcal{F}$ be a finite subset of $A / I$ and $\epsilon>0$. For each $x \in \mathcal{F}$ choose an $x_{1} \in A$ such that $x_{1}+I=x$ and denote by $\mathcal{F}_{1}$ the subset consisting of such $x_{1}$ with 
$x \in \mathcal{F}$. For $\left(\mathcal{F}_{1}, \epsilon\right)$ we obtain $\left(\mathcal{G}_{1}, \delta\right)$ as in Property 2.9. Denoting by $p$ the projection of $D$ onto $B$, we set $\mathcal{G}=\left\{p(x) \mid x \in \mathcal{G}_{1}\right\}$. Then it is easy to check that $(\mathcal{G}, \delta)$ satisfies the requires properties for $(\mathcal{F}, \epsilon)$.

To show (5) note, by $(4)$, that $\left(A_{n}\right)$ can be regarded as an increasing sequence in $\mathcal{C}$. Then for any finite subset $\mathcal{F}$ of $\overline{\cup_{n} A_{n}}$ we find $A_{n}$ which almost contains $\mathcal{F}$. Hence this is immediate.

\section{Homogeneous $C^{*}$-algebras}

In this section we will show that the $C^{*}$-algebras of the form $C \otimes M_{n}$ have Property 2.9, where $C$ is a unital abelian $C^{*}$-algebra. Then it will follow by 2.11 that any approximately homogeneous $C^{*}$-algebra has Property 2.9. Furthermore we will attempt to prove that some subhomogeneous $C^{*}$-algebras have Property 2.9.

The following is well-known.

Lemma 3.1 For any $\epsilon>0$ there is a $\delta>0$ satisfying: If $A$ is a $C^{*}$-algebra and $A_{+1}=$ $\{x \in A \mid x \geq 0,\|x\| \leq 1\}$, then that $\|x-y\|<\delta$ implies that $\left\|x^{1 / 2}-y^{1 / 2}\right\|<\epsilon$ for any $x, y \in A_{+1}$.

Proof. For any $\epsilon>0$ there is a real-valued polynomial $p(t)$ with $p(0)=0$ such that $\left|t^{1 / 2}-p(t)\right|<\epsilon, t \in[0,1]$. If $p(t)=\sum_{i=1}^{n} a_{i} t^{i}$, set $C=\sum_{i}\left|a_{i}\right| i$. Then for $x, y \in A_{+1}$ we have that $\|p(x)-p(y)\| \leq C\|x-y\|$. Hence for $x, y \in A_{+1}$ the condition that $\|x-y\|<\epsilon / C$ implies that $\left\|x^{1 / 2}-y^{1 / 2}\right\|<3 \epsilon$.

Lemma 3.2 For any $\epsilon>0$ and $n \in \mathbf{N}$ there exists a $\delta>0$ satisfying: If $\xi_{1}, \xi_{2}, \ldots, \xi_{n}$ are vectors in a Hilbert space $\mathcal{H}$ with $\operatorname{dim}(\mathcal{H}) \geq n$ and $c=\left(c_{i j}\right)$ is an $n \times n$ matrix such that $\sum_{i=1}^{n}\left\|\xi_{i}\right\|^{2} \leq 1, c \geq 0$, and

$$
\left|c_{i j}-\left\langle\xi_{i}, \xi_{j}\right\rangle\right|<\delta, \quad i, j=1,2, \ldots, n,
$$

then there exist vectors $\eta_{1}, \eta_{2}, \ldots, \eta_{n}$ in $\mathcal{H}$ such that

$$
\begin{aligned}
\left\langle\eta_{i}, \eta_{j}\right\rangle & =c_{i j}, \quad i, j=1,2, \ldots, n \\
\left\|\xi_{i}-\eta_{i}\right\| & <\epsilon, \quad i=1,2, \ldots, n .
\end{aligned}
$$

Proof. Let $d_{i j}=\left\langle\xi_{i}, \xi_{j}\right\rangle$. Then the $n \times n$ matrix $d=\left(d_{i j}\right)$ satisfies that $d \geq 0$ and $\|d\| \leq 1$. The condition that $\left|c_{i j}-d_{i j}\right|<\delta$ for all $i, j$ implies that $\|c-d\| \leq n \delta$.

If $d$ is strictly positive, then define $\eta_{i}=\sum_{k=1}^{n}\left(c^{1 / 2} d^{-1 / 2}\right)_{i k} \xi_{k}$. Then by computation

$$
\left\langle\eta_{i}, \eta_{j}\right\rangle=\sum_{k, \ell}\left(c^{1 / 2} d^{-1 / 2}\right)_{i k}{\overline{\left(c^{1 / 2} d^{-1 / 2}\right)}}_{j \ell} d_{k \ell}=c_{i j}
$$


and

$$
\left\|\eta_{i}-\xi_{i}\right\|^{2}=\left(c^{1 / 2}-d^{1 / 2}\right)_{i i}^{2} \leq\left\|c^{1 / 2}-d^{1 / 2}\right\|^{2} .
$$

By the previous lemma one can choose $\delta>0$ so small that $\left\|c^{1 / 2}-d^{1 / 2}\right\|<\epsilon$ follows from $\|c-d\| \leq n \delta$ and $\|d\| \leq 1$.

In general let $\mathcal{L}$ be a subspace of $\mathcal{H}$ such that $\mathcal{L} \ni \xi_{i}$ for all $i$ and $\operatorname{dim} \mathcal{L}=n$. Then there is a sequence $\left(\xi_{k 1}, \xi_{k 2}, \ldots, \xi_{k n}\right)$ of bases of $\mathcal{L}$ such that $\left\|\xi_{k i}-\xi_{i}\right\| \rightarrow 0$ as $k \rightarrow \infty$. For each $\left(\xi_{k 1}, \xi_{k 2}, \ldots, \xi_{k n}\right)$ one can apply the previous argument to produce vectors $\eta_{k 1}, \eta_{k 2}, \ldots, \eta_{k n}$ in $\mathcal{L}$. By using the compactness argument for the limit of $k$ to infinity one obtains vectors $\eta_{1}, \eta_{2}, \ldots, \eta_{n}$ in $\mathcal{L}$ such that $\left\langle\eta_{i}, \eta_{j}\right\rangle=c_{i j}$ and $\left\|\eta_{i}-\xi_{i}\right\|^{2}=\left(c^{1 / 2}-d^{1 / 2}\right)_{i i}^{2}$. This completes the proof.

Lemma 3.3 For any $\epsilon>0$ and $n \in \mathbf{N}$ there exists $a \delta>0$ satisfying: Given sequences $\left(\xi_{1}, \xi_{2}, \ldots, \xi_{n}\right)$ and $\left(\eta_{1}, \eta_{2}, \ldots, \eta_{n}\right)$ of vectors in a Hilbert space $\mathcal{H}$ such that $\sum_{i}\left\|\xi_{i}\right\|^{2} \leq 1$, $\sum_{i}\left\|\eta_{i}\right\|^{2} \leq 1$, and

$$
\left|\left\langle\xi_{i}, \xi_{j}\right\rangle-\left\langle\eta_{i}, \eta_{j}\right\rangle\right|<\delta, \quad i, j=1,2, \ldots, n,
$$

there is a unitary $U$ on $\mathcal{H}$ such that

$$
\left\|U \xi_{i}-\eta_{i}\right\|<\epsilon, \quad i=1,2, \ldots, n .
$$

Proof. If $\operatorname{dim} \mathcal{H} \geq n$, then choose $\delta>0$ as in the previous lemma and find a sequence $\left(\zeta_{1}, \zeta_{2}, \ldots, \zeta_{n}\right)$ in $\mathcal{H}$ such that $\left\langle\zeta_{i}, \zeta_{j}\right\rangle=\left\langle\xi_{i}, \xi_{j}\right\rangle$ and $\left\|\zeta_{i}-\eta_{i}\right\|<\epsilon$. Then we can find a unitary $U$ on $\mathcal{H}$ such that $U \xi_{i}=\zeta_{i}$ for $i=1,2, \ldots, n$, which satisfies the required properties.

If $m=\operatorname{dim} \mathcal{H}<n$, we first choose $m$ vectors from $\left(\xi_{1}, \xi_{2}, \ldots, \xi_{n}\right)$. Pick up $\xi_{i_{1}}$ with $\left\|\xi_{i_{1}}\right\|=\max _{j}\left\|\xi_{j}\right\|$. Let $P_{1}$ be the projection onto the subspace spanned by $\xi_{i_{1}}$ and pick up $\xi_{i_{2}}$ with $\left\|\left(1-P_{1}\right) \xi_{i_{2}}\right\|=\max _{j}\left\|\left(1-P_{1}\right) \xi_{j}\right\|$. Let $P_{2}$ be the projection onto the subspace spanned by $\xi_{i_{1}}, \xi_{i_{2}}$ and pick up $\xi_{i_{3}}$ with $\left\|\left(1-P_{2}\right) \xi_{i_{3}}\right\|=\max _{j}\left\|\left(1-P_{2}\right) \xi_{j}\right\|$. Repeating this process we obtain $\xi_{i_{1}}, \xi_{i_{2}}, \ldots, \xi_{i_{m}}$, which we assume are all different as we may. If $j \notin I=\left\{i_{1}, i_{2}, \ldots, i_{m}\right\}$, then there are $c_{j}^{k} \in \mathbf{C}$ for $k \in I$ such that

$$
\xi_{j}=\sum_{k \in I} c_{j}^{k} \xi_{k}
$$

From the construction of $I$ we can assume that $\left|c_{j}^{k}\right| \leq 1$. (If $\xi_{k}, k \in I$ are linearly independent, then of course $\left|c_{j}^{k}\right| \leq 1$ automatically.) If $\left|\left\langle\xi_{i}, \xi_{j}\right\rangle-\left\langle\eta_{i}, \eta_{j}\right\rangle\right|<\delta$ for all $i, j$, then $\left\|\eta_{j}-\sum_{k \in I} c_{j}^{k} \eta_{k}\right\|^{2} \leq(m+1)^{2} \delta$. Thus if we define a unitary $U$ on $\mathcal{H}$ by requiring that $\left\|U \xi_{k}-\eta_{k}\right\|<\epsilon, k \in I$, we obtain that for $j \notin I$,

$$
\left\|U \xi_{j}-\eta_{j}\right\| \leq \sum_{k \in I}\left|c_{j}^{k}\right|\left\|U \xi_{k}-\eta_{k}\right\|+\left\|\eta_{j}-\sum_{k \in I} c_{j}^{k} \eta_{k}\right\| \leq m \epsilon+(m+1)^{2} \delta .
$$

This completes the proof. 
Lemma 3.4 Any matrix algebra $M_{n}$ has Property 2.9.

Proof. Let $B$ be a $C^{*}$-algebra containing $A=M_{n}$ with $1_{B}=1_{A}$. Let $\left(e_{i j}\right)$ be the set of matrix units of $A=M_{n}$. Since $A$ is spanned by the matrix units, we may take $\left\{e_{i j} \mid i, j=1,2, \ldots, n\right\}$ for both $\mathcal{F}$ and $\mathcal{G}$ in Property 2.9. Let $\epsilon>0$ and let $\delta>0$ be the $\delta$ for $\epsilon n^{-1 / 2}$ in place of $\epsilon$ in Lemma 3.3 .

Let $\pi$ be an irreducible representation of $B$ on $\mathcal{H}$ and let $\xi, \eta$ be unit vectors in $\mathcal{H}$ such that

$$
\left|\left\langle\pi\left(e_{i j}\right) \xi, \xi\right\rangle-\left\langle\pi\left(e_{i j}\right) \eta, \eta\right\rangle\right|<\delta
$$

for all $i, j$. We apply Lemma 3.3 to the sequences $\left(\pi\left(e_{1 j}\right) \xi\right)$ and $\left(\pi\left(e_{1 j}\right) \eta\right)$ and obtain a unitary $U$ on the subspace $\mathcal{L}$ spanned by $\left(\pi\left(e_{1 j}\right) \xi\right)$ and $\left(\pi\left(e_{1 j}\right) \eta\right)$ such that $\| U \pi\left(e_{1 j}\right) \xi-$ $\pi\left(e_{1 j}\right) \eta \|<\epsilon n^{-1 / 2}$. Since $U=e^{i H}$ with $\|H\| \leq \pi$ we choose a self-adjoint $h=h^{*} \in e_{11} B e_{11}$ such that $\|h\| \leq \pi$ and $\pi(h) \mid \mathcal{L}=H$. Define a unitary $u_{t}$ for each $t \in[0,1]$ by

$$
u_{t}=\sum_{i} e_{i 1} e^{\sqrt{-1} t h} e_{1 i}
$$

Then it follows that $u_{t} \in B \cap A^{\prime}$. By computation

$$
\begin{aligned}
\left\|\pi\left(u_{1}\right) \xi-\eta\right\|^{2} & =\left\|\sum_{i}\left\{\pi\left(e_{i 1}\right) U \pi\left(e_{1 i}\right) \xi-\pi\left(e_{i i}\right) \eta\right\}\right\|^{2} \\
& =\sum_{i}\left\|U \pi\left(e_{1 i}\right) \xi-\pi\left(e_{1 i}\right) \eta\right\|^{2} \\
& <\epsilon^{2} .
\end{aligned}
$$

Thus $\left\|\pi\left(u_{1}\right) \xi-\eta\right\|<\epsilon$. Since $u_{t} \in B \cap A^{\prime}$, this completes the proof.

Proposition 3.5 If $A$ is a unital $C^{*}$-algebra satisfying Property 2.9 and $C$ is a unital commutative $C^{*}$-algebra, then $A \otimes C$ satisfies Property 2.9

Since any unital commutative $C^{*}$-algebra is an inductive limit of quotients of $C\left(\mathbf{T}^{n}\right)$, we may assume that $C=C(\mathbf{T})$ in the above proposition by 2.11. This follows essentially from the following lemma.

Lemma 3.6 For any small $\epsilon>0$ and $\epsilon^{\prime}>0$ there exist $a \delta>0$ and $n \in \mathbf{N}$ satisfying: Let $U$ be a unitary operator on a Hilbert space $\mathcal{H}$ with $E$ its spectral measure and let $\xi, \eta$ be two unit vectors in $\mathcal{H}$. If

$$
\left|\left\langle U^{k} \xi, \xi\right\rangle-\left\langle U^{k} \eta, \eta\right\rangle\right|<\delta
$$

for $k=0, \pm 1, \ldots, \pm n$, there exists a sequence $\left(t_{1}, t_{2}, \ldots, t_{m}\right)$ of points in $\mathbf{T}$ such that $t_{i-1}<t_{i}$ in the cyclic order with $t_{0}=t_{m}, \epsilon / 2<\operatorname{dist}\left(t_{i-1}, t_{i}\right)<3 \epsilon / 2$ and

$$
\begin{aligned}
& \left\|E E\left(t_{i-1}, t_{i}\right) \xi\right\|^{2}-\left\|E\left(t_{i-1}, t_{i}\right) \eta\right\|^{2} \mid<2 \epsilon^{\prime}, \\
& \left\|E\left(t_{i}-\gamma / 2, t_{i}+\gamma / 2\right) \xi\right\|^{2}<\epsilon^{\prime}, \quad\left\|E\left(t_{i}-\gamma / 2, t_{i}+\gamma / 2\right) \eta\right\|^{2}<\epsilon^{\prime},
\end{aligned}
$$

where $\gamma=\epsilon \epsilon^{\prime} / 4$ and the total length of $\mathbf{T}$ is normalized to be 1 . 
Proof. Let $\gamma=\epsilon \epsilon^{\prime} / 4$. For any interval $I$ of $\mathbf{T}=\mathbf{R} / \mathbf{Z}$ of length $\epsilon / 2$, there is a $t \in I$ such that both $\|E(t+(-\gamma / 2, \gamma / 2]) \xi\|^{2}$ and $\|E(t+(-\gamma / 2, \gamma / 2]) \eta\|^{2}$ are smaller than $\epsilon^{\prime}$. Otherwise

$$
\langle E(t+(-\gamma / 2, \gamma / 2]) \xi, \xi\rangle+\langle E(t+(-\gamma / 2, \gamma / 2]) \eta, \eta\rangle \geq \epsilon^{\prime}
$$

for any $t \in I$, which implies that

$$
\langle E(J) \xi, \xi\rangle+\langle E(J) \eta, \eta\rangle>2,
$$

for an interval $J$ including $I$, a contradiction. Thus there is a sequence $\left(t_{1}, t_{2}, \ldots, t_{m}\right)$ of points in $\mathbf{T}$ such that $t_{i-1}<t_{i}$ in the cyclic order and $\epsilon / 2<\operatorname{dist}\left(t_{i-1}, t_{i}\right)<3 \epsilon / 2$ with $t_{0}=t_{m}$, and $\left\|E\left(J_{i}\right) \xi\right\|^{2}<\epsilon^{\prime}$ and $\left\|E\left(J_{i}\right) \eta\right\|^{2}<\epsilon^{\prime}$ for $J_{i}=t_{i}+(-\gamma / 2, \gamma / 2]$.

Let $f$ be a $C^{\infty}$-function on $\mathbf{T}$ such that $0 \leq f \leq 1, \operatorname{supp} f \subset[0,1 / 4]$, and $f=1$ on $[\gamma / 2,1 / 4-\gamma / 2]$. If $\xi, \eta$ satisfy the condition in the statement for a sufficiently small $\delta$ and for a sufficiently large $n$, it follows that for any product $g$ of two translates of $f$,

$$
|\langle g(U) \xi, \xi\rangle-\langle g(U) \eta, \eta\rangle|<\epsilon^{\prime} .
$$

We construct a function $f_{i}$, as the product of two translates of $f$, such that $\operatorname{supp} f_{i} \subset$ $\left[t_{i-1}, t_{i}\right]$ and $f_{i}=1$ on $\left[t_{i-1}+\gamma / 2, t_{i}-\gamma / 2\right]$. Since $\left|\left\langle f_{i}(U) \xi, \xi\right\rangle-\left\langle f_{i}(U) \eta, \eta\right\rangle\right|<\epsilon^{\prime}$, and $\left\|E\left(J_{i}\right) \xi\right\|^{2}<\epsilon^{\prime}$ etc., we have that for any $i$,

$$
\left|\left\|E\left(t_{i-1}, t_{i}\right) \xi\right\|^{2}-\left\|E\left(t_{i-1}, t_{i}\right) \eta\right\|^{2}\right|<2 \epsilon^{\prime} .
$$

This completes the proof.

Proof of Proposition 3.5 By the following lemma, for any finite subset $\mathcal{F}$ of $A$ and $\epsilon>0$ we have a finite subset $\mathcal{G}_{A}$ of $A$ and $\delta^{\prime}>0$ satisfying: Let $B$ be a non-unital $C^{*}$-algebra such that $A$ is a unital $C^{*}$-subalgebra of the multiplier algebra $M(B)$ of $B$. If two pure states $\omega_{1}, \omega_{2}$ of $B$ satisfy that $\omega_{1} \sim \omega_{2}$ and $\left|\omega_{1}(x)-\omega_{2}(x)\right|<\delta^{\prime}, x \in \mathcal{G}_{A}$, where $\omega_{i}$ also denotes the natural extension of $\omega_{i}$ to a state on $M(B)$, then there is a continuous path $\left(u_{t}\right)$ in $\mathcal{U}(B)$ such that $u_{t}-1 \in B, u_{0}=1, \omega_{1}=\omega_{2} \operatorname{Ad} u_{1}$, and

$$
\left\|\operatorname{Ad} u_{t}(x)-x\right\|<\epsilon, \quad x \in \mathcal{F} .
$$

We may assume, by replacing $\delta$ by a smaller one, that $\|x\| \leq 1$ for $x \in \mathcal{G}_{A}$ and also that $1 \in \mathcal{G}_{A}$.

Suppose that $B \supset A \otimes C(\mathbf{T})$ with the common unit. Given $\epsilon>0$ and $\epsilon^{\prime}=\epsilon^{3} \delta^{\prime} / 4$ we choose $\delta, n$ as in the previous lemma.

Let $\mathcal{G}=\left\{x z^{k}\left|x \in \mathcal{G}_{A},\right| k \mid \leq n\right\}$, where $z$ is the canonical unitary of $C(\mathbf{T}) \simeq$ $1 \otimes C(\mathbf{T}) \subset A \otimes C(\mathbf{T})$. Let $\pi$ be an irreducible representation of $B$ and $\omega_{1}, \omega_{2}$ be the pure states of $B$ defined through unit vectors $\xi, \eta$ respectively. Suppose that

$$
\left|\omega_{1}(x)-\omega_{2}(x)\right|<\min \left(\delta, \epsilon^{\prime}\right), \quad x \in \mathcal{G} .
$$


For $U=\pi(z)$ we have that

$$
\left|\left\langle U^{k} \xi, \xi\right\rangle-\left\langle U^{k} \eta, \eta\right\rangle\right|<\delta, \quad|k| \leq n .
$$

Then we obtain a sequence $\left(t_{1}, t_{2}, \ldots, t_{m}\right)$ in $\mathbf{T}$ as in 3.6. Let $B_{i}$ be the hereditary $C^{*}$ subalgebra of $B$ generated by $E\left(t_{i-1}, t_{i}\right)$, where $E$ is the spectral measure of $z$. There is a natural unital embedding of $A$ into the multiplier algebra $M\left(B_{i}\right)$. Let $f_{i} \in C(\mathbf{T})$ be a function supported on $\left[t_{i-1}, t_{i}\right]$ as given in the proof of the previous lemma. Then from the proof of 3.6 we may assume that

$$
\left|\omega_{1}\left(x f_{i}(z)\right)-\omega_{2}\left(x f_{i}(z)\right)\right|<\epsilon^{\prime}, \quad x \in \mathcal{G}_{A},
$$

which then implies that

$$
\left|\left\langle x E\left(t_{i-1}, t_{i}\right) \xi, \xi\right\rangle-\left\langle x E\left(t_{i-1}, t_{i}\right) \eta, \eta\right\rangle\right|<2 \epsilon^{\prime}, \quad x \in \mathcal{G}_{A} .
$$

We define constants $c_{i}, d_{i}$ by

$$
d_{i}\left\|E\left(t_{i-1}, t_{i}\right) \xi\right\|=c_{i}\left\|E\left(t_{i-1}, t_{i}\right) \eta\right\|=\min \left(\left\|E\left(t_{i-1}, t_{i}\right) \xi\right\|,\left\|E\left(t_{i-1}, t_{i}\right) \eta\right\|\right),
$$

where $E$ is regarded as the spectral measure of $U=\pi(z)$. If

$$
d_{i}\left\|E\left(t_{i-1}, t_{i}\right) \xi\right\|=c_{i}\left\|E\left(t_{i-1}, t_{i}\right) \eta\right\| \leq \epsilon^{3 / 2},
$$

then we set $u_{i t}=1 \in \mathcal{U}\left(B_{i}\right)$; otherwise for the states on $B_{i}: \varphi_{1}=\left\|E\left(t_{i-1}, t_{i}\right) \xi\right\|^{-2} \omega_{1} \mid B_{i}$ and $\varphi_{2}=\left\|E\left(t_{i-1}, t_{i}\right) \eta\right\|^{-2} \omega_{2} \mid B_{i}$, it follows that

$$
\left|\varphi_{1}(x)-\varphi_{2}(x)\right|<4 \epsilon^{\prime} \epsilon^{-3}=\delta^{\prime}, \quad x \in \mathcal{G}_{A} .
$$

Here we have used the fact that $\left|1-\left\|E\left(t_{i-1}, t_{i}\right) \xi\right\|^{-2}\left\|E\left(t_{i-1}, t_{i}\right) \eta\right\|^{2}\right|<2 \epsilon^{\prime} \epsilon^{-3}$ and $\|x\| \leq 1$ for $x \in \mathcal{G}_{A}$. Hence we obtain a continuous path $\left(u_{i t}\right)$ in $\mathcal{U}\left(B_{i}\right)$ such that $u_{i t}-1 \in B_{i}$, $\left\|\operatorname{Ad} u_{i t}(x)-x\right\|<\epsilon, x \in \mathcal{F}$, and $\pi\left(u_{i 1}\right) d_{i} E\left(t_{i-1}, t_{i}\right) \xi=c_{i} E\left(t_{i-1}, t_{i}\right) \eta$. Then the sum $\left(u_{t}\right)$ of $\left(u_{i t}\right)$ over $i$ defines a continuous path in $\mathcal{U}(B)$ (or regarding each $u_{i t}$ as in $\mathcal{U}(B)$ take the product of these $u_{i t}$ over $\left.i\right)$. Then, since $\operatorname{dist}\left(t_{i-1}, t_{i}\right)<3 \epsilon / 2$, we have that $\left\|u_{t} z-z u_{t}\right\|<3 \pi \epsilon$ and also that

$$
\left\|\operatorname{Ad} u_{t}(x)-x\right\|<\epsilon, \quad x \in \mathcal{F} .
$$

Let $S$ be the set of $i$ with $d_{i}\left\|E\left(t_{i-1}, t_{i}\right) \xi\right\|>\epsilon^{3 / 2}$. Then

$$
\sum_{i \notin S}\left\|d_{i} E\left(t_{i-1}, t_{i}\right) \xi\right\|^{2}<2 \epsilon^{2}
$$

and hence

$$
\sum_{i \in S}\left\|d_{i} E\left(t_{i-1}, t_{i}\right) \xi\right\|^{2}>1-3 \epsilon^{2}
$$


because $\sum_{i}\left\|E\left(\left\{t_{i}\right\}\right) \xi\right\|^{2}<2 \epsilon^{\prime} \epsilon^{-1}<\epsilon^{2} \delta^{\prime} / 2$, which can be assumed to be smaller than $\epsilon^{2}$. Since $\pi\left(u_{1}\right) d_{i} E\left(t_{i-1}, t_{i}\right) \xi=c_{i} E\left(t_{i-1}, t_{i}\right) \eta$, for $i \in S$, we have that

$$
\pi\left(u_{1}\right) \sum_{i \in S} d_{i} E\left(t_{i-1}, t_{i}\right) \xi=\sum_{i \in S} c_{i} E\left(t_{i-1}, t_{i}\right) \eta
$$

This implies that

$$
\left\|\pi\left(u_{1}\right) \xi-\eta\right\|<2 \sqrt{3} \epsilon
$$

This completes the proof.

Lemma 3.7 If $A$ is a unital $C^{*}$-algebra satisfying Property 2.9. Then for any finite subset $\mathcal{F}$ of $A$ and $\epsilon>0$ there is a finite subset $\mathcal{G}$ of $A$ and $\delta>0$ satisfying: If $B$ is a non-unital $C^{*}$-algebra such that $A \subset M(B)$ with common unit and $\pi$ is an irreducible representation of $B$, and $\xi, \eta$ are unit vectors in $\mathcal{H}_{\pi}$ such that if

$$
|\langle\pi(x) \xi, \xi\rangle-\langle\pi(x) \eta, \eta\rangle|<\delta, \quad x \in \mathcal{G},
$$

where $\pi$ also denotes the natural extension of $\pi$ to a representation of $M(B)$, then there is a continuous path $\left(u_{t}\right)_{t \in[0,1]}$ in $\mathcal{U}(B)$ such that $u_{0}=1, \eta=\pi\left(u_{1}\right) \xi, u_{t}-1 \in B$, and

$$
\left\|\operatorname{Ad} u_{t}(x)-x\right\|<\epsilon, \quad x \in \mathcal{F}, t \in[0,1] .
$$

Proof. In the situation as above, for the choice of $(\mathcal{G}, \delta)$ made for Property 2.9, we have a continuous path $\left(u_{t}\right)$ in $\mathcal{U}(M(B))$ such that $u_{0}=1, \eta \in \mathbf{C} \pi\left(u_{1}\right) \xi$ and

$$
\left\|\operatorname{Ad} u_{t}(x)-x\right\|<\epsilon, \quad x \in \mathcal{F}, t \in[0,1] .
$$

By multiplying $\left(u_{t}\right)$ by a suitable continuous function, we may assume that $\eta=\pi\left(u_{1}\right) \xi$.

Let $\left(e_{\iota}\right)$ be an approximate identity of $B$. Since $\left[e_{\iota}, x\right] \in B$ converges to zero in the $\sigma\left(B, B^{*}\right)$ topology for any $x \in M(B)$, we can assume, by taking a net from the convex combinations of $e_{\iota}$ 's if necessary, that $\left[e_{\iota}, x\right]$ converges to zero in norm for $x \in M(B)$; in particular $\left[e_{\iota}, u_{t}\right]$ converges to zero as well as $\left[e_{\iota}, x\right], x \in \mathcal{F}$. Thus we find a sequence $\left(e_{n}\right)$ in $B$ such that $0 \leq e_{n} \leq 1, \pi\left(e_{n}\right) \xi \rightarrow \xi,\left[e_{n}, u_{t}\right] \rightarrow 0,\left[e_{n}, x\right] \rightarrow 0, x \in \mathcal{F}$, and $e_{n} e_{m}-e_{m} \rightarrow 0$ as $n \rightarrow \infty$ for each $m$. Let $\mu \in(0,1)$ be so close to 1 that $\left\|u_{t}-u_{\mu t}\right\|<\epsilon$ for $t \in[0,1]$ and let $k \in \mathbf{N}$ be such that $\left\|u_{\mu^{k} t}-1\right\|<\epsilon$. For a subsequence $\left(n_{i}\right)$ set

$$
z_{t}=u_{t} e_{n_{1}}+u_{\mu t} p_{1}+u_{\mu^{2} t} p_{2}+\cdots+u_{\mu^{k} t} p_{k}+1-e_{n_{k+1}}
$$

where $p_{i}=e_{n_{i+1}}-e_{n_{i}}$. By assuming that $e_{n_{i+1}} e_{n_{i}} \approx e_{n_{i}}$, we have that $p_{i} p_{j} \approx 0$ if $|i-j|>1$. We can also assume that $z_{t}$ almost commutes with $e_{n_{i}}$ and $x \in \mathcal{F}$. Hence $z_{t} p_{i} \approx u_{\mu^{i} t} p_{i}$, $z_{t} e_{n_{1}} \approx u_{t} e_{n_{1}}$, and $z_{t}\left(1-e_{n_{k+1}}\right) \approx 1-e_{n_{k+1}}$, all up to $\epsilon$. Since $1=e_{n_{1}}+\sum_{i=1}^{k} p_{i}+1-e_{n_{k+1}}$ and $p_{i} z_{t}^{*} z_{t} p_{j}$ is arbitrarily close to zero if $|i-j|>1$ for $i, j=0,1, \ldots, k+1$ with $p_{0}=e_{n_{1}}, p_{k+1}=1-e_{n_{k+1}}$, we can conclude that $z_{t}^{*} z_{t} \approx 1$ up to the order of $\epsilon$. (For example $\left\|z_{t}^{*} z_{t}-1\right\|=\left\|\sum_{i=0}^{k+1}\left(z_{t}^{*} z_{t}-1\right) p_{i}\right\| \leq\left\|\sum\left(z_{t}^{*} z_{t}-1\right) p_{2 i}\right\|+\left\|\sum\left(z_{t}^{*} z_{t}-1\right) p_{2 i+1}\right\| \lesssim$ 
$\left.2 \max _{i}\left\|\left(z_{t}^{*} z_{t}-1\right) p_{i}\right\|.\right)$ Hence $z_{t}$ is close to a unitary in $B+1$ up to the order of $\epsilon$. By assuming that $\pi\left(e_{n_{1}}\right) \xi \approx \xi$, we have that $\pi\left(z_{1}\right) \xi \approx \eta$. In this way we can construct a continuous path $\left(v_{t}\right)$ in $\mathcal{U}(B)$ in a small neighborhood of $\left(z_{t}\right)$ (of order $\epsilon$ ) such that $\pi\left(v_{1}\right) \xi \approx \eta, v_{t}-1 \in B$, and $\left\|\left[v_{t}, x\right]\right\| \approx 0$ up to the order of $\epsilon$ for $x \in \mathcal{F}$. This completes the proof.

For $n=1,2, \ldots$ let $D_{n}$ denote the dimension drop $C^{*}$-algebra:

$$
D_{n}=\left\{x \in C\left([0,1] ; M_{n}\right) \mid x(0), x(1) \in \mathbf{C} 1_{n}\right\} .
$$

Lemma 3.8 For any $k, n \in \mathbf{N}, D_{n} \otimes M_{k}$ has Property 2.9.

Proof. For any finite subset $\mathcal{F}$ of $D_{n} \otimes M_{k}$ and $\epsilon>0$, we find an interval $[a, b] \subset(0,1)$ such that if $x \in \mathcal{F}$, then $\|x(t)-x(0)\|<\epsilon$ for any $t \in[0, a]$ and $\|x(t)-x(1)\|<\epsilon$ for any $t \in[b, 1]$. Hence we may suppose that if $x \in \mathcal{F}$, then $x(t)=x(0) \in 1_{n} \otimes M_{k}$ for $t \in[0, a]$ and $x(t)=x(1) \in 1_{n} \otimes M_{k}$ for $t \in[b, 1]$. Replacing $a$ by a smaller one and $b$ by a larger one, we may further suppose that if $x \in \mathcal{F}$, then $x(t)=x(0)$ around $t=a$ and $x(t)=x(1)$ around $t=b$. Denoting by $\mathcal{F} \mid[a, b]$ the subset of $C[a, b] \otimes M_{n} \otimes M_{k}$ obtained by restricting $\mathcal{F}$ to $[a, b]$, we choose $\left(\mathcal{G}_{2}, \delta_{2}\right)$ for $(\mathcal{F} \mid[a, b], \epsilon)$ as in 3.7 . Similarly we choose $\left(\mathcal{G}_{0}, \delta_{0}\right)$ for $(\mathcal{F} \mid[0, a], \epsilon)$ with $\mathcal{F} \mid[0, a]$ as a subset of $1 \otimes M_{k}$ and $\left(\mathcal{G}_{1}, \delta_{1}\right)$ for $(\mathcal{F} \mid[b, 1], \epsilon)$ with $\mathcal{F} \mid[b, 1]$ as a subset of $1 \otimes M_{k}$ by using [3.7. We may assume that $\delta_{0}=\delta_{1}=\delta_{2} \equiv \delta$ and $\|x\| \leq 1$ for $x \in \mathcal{G}_{0} \cup \mathcal{G}_{1} \cup \mathcal{G}_{2}$. Furthermore we may assume that if $x \in \mathcal{G}_{2}$, then $x(t)=x(a)$ around $t=a$ in $[a, b]$ and $x(t)=x(b)$ around $t=b$ in $[a, b]$. For $x \in \mathcal{G}_{2}$, we set

$$
\bar{x}(t)= \begin{cases}x(b) & t>b \\ x(t) & a \leq t \leq b \\ x(a) & t<a\end{cases}
$$

and define $\overline{\mathcal{G}}_{2}=\left\{\bar{x} \mid x \in \mathcal{G}_{2}\right\} \cup\{1\} \subset C[0,1] \otimes M_{n} \otimes M_{k}$. If $0<a^{\prime}<a$ and $b<b^{\prime}<1$, we can choose $\left(\overline{\mathcal{G}} \mid\left[a^{\prime}, b^{\prime}\right], \delta\right)$ as the $(\mathcal{G}, \delta)$ for $\left(\mathcal{F} \mid\left[a^{\prime}, b^{\prime}\right], \epsilon\right)$ in 3.7; because there is an isomorphism of $C[a, b] \otimes M_{n} \otimes M_{k}$ onto $C\left[a^{\prime}, b^{\prime}\right] \otimes M_{n} \otimes M_{k}$ which sends $\mathcal{F} \mid[a, b]$ onto $\mathcal{F} \mid\left[a^{\prime}, b^{\prime}\right]$ and $\mathcal{G}_{2}=\overline{\mathcal{G}}_{2} \mid[a, b]$ onto $\overline{\mathcal{G}}_{2} \mid\left[a^{\prime}, b^{\prime}\right]$ respectively.

Let $N \in \mathbf{N}$ be such that $N \delta \epsilon^{2}>16$, and let $\epsilon^{\prime}>0$ be such that $N \epsilon^{\prime}<\min (a, 1-b)$. Let

$$
I_{k}=\left(a-(k+1) \epsilon^{\prime}, a-k \epsilon^{\prime}\right], \quad J_{k}=\left(b+k \epsilon^{\prime}, b+(k+1) \epsilon^{\prime}\right]
$$

for $k=0,1, \ldots, N-1$. Let $f_{k} \in C[0,1]$ be such that $0 \leq f_{k} \leq 1, \operatorname{supp} f_{k} \subset(a-(k+$ 1) $\left.\epsilon^{\prime}, b+(k+1) \epsilon^{\prime}\right]$, and $f_{k}(t)=1$ for $t \in\left[a-k \epsilon^{\prime}, b+k \epsilon^{\prime}\right]$ and let $f_{0 k}=\left(1-f_{k}\right) \chi_{[0, a]} \in C[0,1]$ and $f_{1 k}=\left(1-f_{k}\right) \chi_{[b, 1]} \in C[0,1]$. Finally we define a subset $\mathcal{G}$ of $D_{n} \otimes M_{k}$ as the union of $\left\{f_{k} x \mid x \in \overline{\mathcal{G}}_{2}, k=0,1, \ldots, N-1\right\},\left\{f_{0 k} x \mid x \in \mathcal{G}_{0}\right.$ or $\left.x=1 ; k=0, \ldots, N-1\right\}$, and $\left\{f_{1 k} x \mid x \in \mathcal{G}_{1}\right.$ or $\left.x=1 ; k=0, \ldots, N-1\right\}$.

Let $B$ be a $C^{*}$-algebra containing $D_{n} \otimes M_{k}$ as a unital $C^{*}$-subalgebra and let $\pi$ be an irreducible representation of $B$. Let $\xi, \eta$ be unit vectors in $\mathcal{H}_{\pi}$ such that

$$
|\langle\pi(x) \xi, \xi\rangle-\langle\pi(x) \eta, \eta\rangle|<\delta \epsilon^{2} / 4, \quad x \in \mathcal{G} .
$$


We first choose $k$ such that

$$
s_{k} \equiv\left\|\chi_{I_{k}} \xi\right\|^{2}+\left\|\chi_{I_{k}} \eta\right\|^{2}+\left\|\chi_{J_{k}} \xi\right\|^{2}+\left\|\chi_{J_{k}} \eta\right\|^{2}<\delta \epsilon^{2} / 4,
$$

where $\chi_{I_{k}}$ is the characteristic function of $I_{k}$ regarded as an element of $\left(D_{n} \otimes M_{k}\right)^{* *} \subset B^{* *}$, which is also identified with $\pi^{* *}\left(\chi_{I_{k}}\right)$ etc. (Otherwise $s_{k} \geq \delta \epsilon^{2} / 4$ for all $k$ leads us to a contradiction because $N \delta \epsilon^{2}>16$.)

Let $a^{\prime}=a-(k+1) \epsilon^{\prime}$ and $b^{\prime}=b+(k+1) \epsilon^{\prime}$, and let $e=\chi_{\left(a^{\prime}, b^{\prime}\right)} \in B^{* *}$ and $B_{2}$ the hereditary $C^{*}$-subalgebra of $B$ corresponding to $e$. Since $\left|\left\langle\pi\left(f_{k} x\right) \xi, \xi\right\rangle-\left\langle\pi\left(f_{k} x\right) \eta, \eta\right\rangle\right|<$ $\delta \epsilon^{2} / 4, \quad x \in \overline{\mathcal{G}}_{2}$, we have that

$$
|\langle e x \xi, \xi\rangle-\langle e x \eta, \eta\rangle|<\delta \epsilon^{2} / 2, \quad x \in \overline{\mathcal{G}}_{2},
$$

where we have omitted $\pi$ and $\pi^{* *}$. If $\|e \xi\|>\epsilon$ (and $\epsilon, \delta$ are sufficiently small), then it follows that

$$
\left|\left\langle\pi_{2}(x) \xi_{2}, \xi_{2}\right\rangle-\left\langle\pi_{2}(x) \eta_{2}, \eta_{2}\right\rangle\right|<\delta, \quad x \in \overline{\mathcal{G}}_{2} \mid\left[a^{\prime}, b^{\prime}\right],
$$

where $\pi_{2}$ is the irreducible representation of $B_{2}$ on $e \mathcal{H}_{\pi}$ obtained by restricting $\pi$, and $\xi_{2}=\|e \xi\|^{-1} e \xi$ and $\eta_{2}=\|e \eta\|^{-1} e \eta$. Note that $C\left[a^{\prime}, b^{\prime}\right] \otimes M_{n} \otimes M_{k}$ is regarded as a subalgebra of the multiplier algebra of $B_{2}$. Hence by 3.7 we can find a continuous path $\left(v_{t}\right)$ in $\mathcal{U}\left(B_{2}\right)$ such that $v_{0}=1, v_{t}-1 \in B_{2}, \pi_{2}\left(v_{1}\right) \xi_{2}=\eta_{2}$, and $\left\|\operatorname{Ad} v_{t}(x)-x\right\|<\epsilon, x \in \mathcal{F} \mid\left[a^{\prime}, b^{\prime}\right]$. If $\|e \xi\| \leq \epsilon$, we set $v_{t}=1$. In any case we have that $\pi_{2}\left(v_{1}\right) \xi_{2} \approx \eta_{2}$ up to $\epsilon$.

Let $B_{0}$ be the hereditary $C^{*}$-subalgebra of $B$ corresponding to $e_{0}=\chi_{\left[0, a^{\prime}\right)}$. If $\left\|e_{0} \xi\right\|>\epsilon$, it follows that

$$
\left|\left\langle\pi_{0}(x) \xi_{0}, \xi_{0}\right\rangle-\left\langle\pi_{0}(x) \eta_{0}, \eta_{0}\right\rangle\right|<\delta, \quad x \in \mathcal{G}_{0},
$$

where $\pi_{0}$ is the irreducible representation of $B_{0}$ on $e_{0} \mathcal{H}_{\pi}$ obtained by restricting $\pi$, and $\xi_{0}=\left\|e_{0} \xi\right\|^{-1} e_{0} \xi$ and $\eta_{0}=\left\|e_{0} \eta\right\|^{-1} e_{0} \eta$. Hence we obtain a continuous path $\left(v_{0 t}\right)$ in $\mathcal{U}\left(B_{0}\right)$ such that $v_{0,0}=1, v_{0 t}-1 \in B_{0}, \pi_{0}\left(v_{0,1}\right) \xi_{0}=\eta_{0}$, and $\left\|\operatorname{Ad} v_{0 t}(x)-x\right\|<\epsilon, x \in \mathcal{F} \mid\left[0, a^{\prime}\right]$. If $\left\|e_{0} \xi\right\| \leq \epsilon$, then we set $v_{0 t}=1$. As before in any case we have that $\pi_{0}\left(v_{0,1}\right) \xi_{0} \approx \eta_{0}$ up to $\epsilon$.

In a similar way we obtain a continuous path $\left(v_{1 t}\right)$ in $\mathcal{U}\left(B_{1}\right)$ with $B_{1}$ the hereditary $C^{*}$-subalgebra of $B$ corresponding to $\chi_{\left(b^{\prime}, 1\right]}$ with similar properties to the above. Since $\left\|\left(e+e_{0}+e_{1}\right) \xi\right\|^{2}=\|e \xi\|^{2}+\left\|e_{0} \xi\right\|^{2}+\left\|e_{1} \xi\right\|^{2} \approx 1$ up to $\epsilon^{2}$, we get the desired path in $\mathcal{U}(B)$ by summing these paths $\left(v_{t}\right),\left(v_{0 t}\right)$, and $\left(v_{1 t}\right)$.

We recall that $A$ is called an AH algebra (or an approximately homogeneous $C^{*}$ algebra) if $A$ is an inductive limit of $C^{*}$-algebras of the form $\oplus_{i=1}^{k} e_{i}\left(C_{i} \otimes M_{n_{i}}\right) e_{i}$, where $C_{i}$ is a unital commutative $C^{*}$-algebra and $e_{i}$ is a projection of $C_{i} \otimes M_{n_{i}}$. The class of $\mathrm{AH}$ algebras include UHF algebras and AF algebras.

More generally, a $C^{*}$-algebra $A$ is called an ASH algebra (or an approximately subhomogeneous $C^{*}$-algebra) if $A$ is an inductive limit of $C^{*}$-algebras of the form

$$
\bigoplus_{i=1}^{k} e_{i}\left(C_{i} \otimes M_{n_{i}}\right) e_{i} \oplus \bigoplus_{j=1}^{r} D_{m_{j}} \otimes M_{k_{j}},
$$


where the first term is given as above. Some classes of ASH algebras are classified in terms of K theory (see, e.g., [6, 5]); in particular a certain class of ASH algebras of real rank zero is classified by Dadarlat and Gong [5]. The following follows from 3.5, 3.8, and 2.11 (3) and (5).

Theorem 3.9 Any ASH algebra (in the above sense) has Property 2.9.

\section{Crossed products of $\mathrm{AF}$ algebras by $\mathrm{Z}$}

Let $A$ be an AF $C^{*}$-algebra. If $\alpha \in \overline{\operatorname{Inn}}(A)=\operatorname{AInn}(A)$ has the Rohlin property, then $A \times{ }_{\alpha} \mathbf{Z}$ is a simple AT algebra and hence, as being an AH algebra, satisfies Property 2.9. More generally we have the following:

Theorem 4.1 Let $A$ be an $A F C^{*}$-algebra and $\alpha \in \operatorname{Aut}(A)$. If the crossed product $A \times_{\alpha} \mathbf{Z}$ is simple, then $A \times_{\alpha} \mathbf{Z}$ has Property 2.9 .

Proof. If $A \times{ }_{\alpha} \mathbf{Z}$ is isomorphic to the compact operators, then this follows from 3.9. Hence we may assume that $A \times_{\alpha} \mathbf{Z}$ is not of type $\mathrm{I}$. (This assumption will be used only at the end of the proof.)

Since $A$ is an $\operatorname{AF} C^{*}$-algebra, there is an increasing sequence $\left(A_{n}\right)$ of finite-dimensional $C^{*}$-subalgebras of $A$ such that $A=\overline{\cup_{n} A_{n}}$. By passing to a subsequence of $\left(A_{n}\right)$ we find a sequence $\left(u_{n}\right)$ in $\mathcal{U}(A)$ such that $u_{2 n+1} \in A \cap A_{2 n}^{\prime}$ with $A_{0}=0, u_{2 n} \in A \cap$ $\operatorname{Ad}\left(u_{2 n-1} u_{2 n-2} \cdots u_{1}\right) \alpha\left(A_{2 n-1}\right)^{\prime},\left\|u_{n}-1\right\|<2^{-n}$, and

$$
\operatorname{Ad} u_{1} \alpha\left(A_{1}\right) \subset A_{2} \subset \operatorname{Ad} u_{3} u_{2} u_{1} \alpha\left(A_{3}\right) \subset A_{4} \subset \cdots .
$$

By replacing $\alpha$ by $\operatorname{Ad} u \alpha$ with $u=\lim _{n} u_{n} u_{n-1} \cdots u_{1}$ and passing to a subsequence of $\left(A_{n}\right)$, we assume that

$$
\alpha^{ \pm 1}\left(A_{n}\right) \subset A_{n+1}
$$

for all $n$. We denote by $1_{n}$ the unit of $A_{n}$. It is well-known that the crossed product $A \times{ }_{\alpha} \mathbf{Z}$ remains the same for this inner perturbation of $\alpha$.

Let $u$ denote the canonical unitary in $A \times_{\alpha} \mathbf{Z}$, i.e., $A \times_{\alpha} \mathbf{Z}$ is generated by $A$ and $u$ with relation that $u x=\alpha(x) u, x \in A$.

Let $\mathcal{F}$ be a finite subset of $A \times{ }_{\alpha} \mathbf{Z}$ and $\epsilon>0$. By taking a smaller $\epsilon>0$ if necessary, we can assume that $\mathcal{F}$ is $\left\{e_{s, i j}^{(k)} \mid i, j, s\right\} \cup\left\{1_{k} u 1_{k}\right\}$, where $\left(e_{s, i j}^{(k)}\right)$ is a family of matrix units for $A_{k}$ and $s \in\left\{1,2, \ldots, N_{k}\right\}$ corresponds to each direct summand of $A_{k}$.

Assume that $n \in \mathbf{N}$ is so large that $2 / n<\epsilon$. We take for $\mathcal{G}$ the set

$$
\left\{1_{k} e_{s, i j}^{(k+2 n)} u^{m} e_{t, a b}^{(k+2 n)} 1_{k} \mid s, i, j, t, a, b ; m=0, \pm 1, \pm 2, \ldots, \pm 2 n\right\},
$$

where we assume that $1_{k}$ is the sum of some of $e_{t, a a}^{(k+2 n)}$. Let $B$ be a $C^{*}$-algebra such that $B \supset A \times{ }_{\alpha} \mathbf{Z}$ and let $\pi$ be an irreducible representation of $B$ with two unit vectors $\xi, \eta$ which define pure states $\omega_{1}, \omega_{2}$ of $B$ respectively. Let $\delta^{\prime}>0$ and suppose that

$$
|\langle\pi(x) \xi, \xi\rangle-\langle\pi(x) \eta, \eta\rangle|<\delta^{\prime}, \quad x \in \mathcal{G} .
$$


We may assume that $\delta^{\prime}$ is so small that either, $\left\|1_{k} \xi\right\|<\epsilon$ and $\left\|1_{k} \eta\right\|<\epsilon$, or otherwise

$$
\left|\left\langle u^{m} e_{s, i j}^{(k+2 n)} \xi_{1}, u^{\ell} e_{t, a b}^{(k+2 n)} \xi_{1}\right\rangle-\left\langle u^{m} e_{s, i j}^{(k+2 n)} \eta_{1}, u^{\ell} e_{t, a b}^{(k+2 n)} \eta_{1}\right\rangle\right|<\delta
$$

for $m, \ell=0,1,2, \ldots, n$ and for some prescribed $\delta>0$, where we have omitted $\pi$, and $\xi_{1}=\left\|1_{k} \xi\right\|^{-1} 1_{k} \xi$ and $\eta_{1}=\left\|1_{k} \eta\right\|^{-1} 1_{k} \eta$.

In the former case we should just take a continuous path $\left(v_{t}\right)$ in $\mathcal{U}\left(\left(1-1_{k}\right) B\left(1-1_{k}\right)\right)$ such that $v_{1}\left(1-1_{k}\right) \xi \approx\left(1-1_{k}\right) \eta$ and set $u_{t}=v_{t}+1_{k}$. Since $u_{t}$ commutes with $\mathcal{F}$ and $u_{1} \xi \approx \eta$, this completes the proof.

In the latter case suppose that the linear space $\mathcal{L}_{\xi_{1}}$ spanned by $u^{m} e_{s, i j}^{k+2 n} 1_{k} \xi_{1}$ with $m=0,1, \ldots, n$ and all possible $s, i, j$ is orthogonal to the space $\mathcal{L}_{\eta_{1}}$ spanned by vectors of the same form with $\eta_{1}$ in place of $\xi_{1}$. If $\delta$ is sufficiently small, then we obtain vectors $\zeta(m, s, i, j)$ in $\mathcal{H}_{\pi} \ominus L_{\xi_{1}}$ for $m=0,1, \ldots, n$ and for all $s, i, j$ with $e_{s, i, j}^{(k+2 n)} 1_{k} \neq 0$ such that

$$
\left\langle u^{m} e_{s, i j}^{(k+2 n)} \xi_{1}, u^{\ell} e_{t, a b}^{(k+2 n)} \xi_{1}\right\rangle=\langle\zeta(m, s, i, j), \zeta(\ell, t, a, b)\rangle
$$

and

$$
\left\|u^{m} e_{s, i j}^{(k+2 n)} 1_{k} \eta-\zeta(m, s, i, j)\right\|<\epsilon^{\prime}
$$

for a small $\epsilon^{\prime}$. Then we can define a projection $E$ on $\mathcal{L}_{\xi_{1}}+\mathcal{L}_{\zeta}$ such that

$$
\begin{aligned}
& E\left(u^{m} e_{s, i j}^{(k+2 n)} 1_{k} \xi+\zeta(m, s, i, j)\right)=0, \\
& E\left(u^{m} e_{s, i j}^{(k+2 n)} 1_{k} \xi-\zeta(m, s, i, j)\right)=u^{m} e_{s, i j}^{(k+2 n)} 1_{k} \xi-\zeta(m, s, i, j) .
\end{aligned}
$$

Then we find an $h=h^{*} \in B$ such that $\|h\|=1$, and $\pi(h) \mid \mathcal{L}_{\xi}+\mathcal{L}_{\zeta}=E$, where $\zeta$ denotes $\sum_{s, i} \zeta(0, s, i, i)$ and $\mathcal{L}_{\zeta}$ is the space spanned by $\zeta(m, s, i, j)$ 's. Define

$$
\bar{h}=\frac{1}{n} \sum_{j=1}^{n-1} \sum_{s} \sum_{i} u^{-j} e_{s, i 1}^{(k+n)} h e_{s, 1 i}^{(k+n)} u^{j} .
$$

Then $\|[u, \bar{h}]\|<2 / n<\epsilon$. Since $\sum_{s} \sum_{i} e_{s, i 1}^{(k+n)} h e_{s, 1 i}^{(k+n)} \in A \cap A_{k+n}^{\prime}$ and $u^{j} A_{k} u^{-j} \subset A_{k+|j|}$, we have that $\bar{h} \in B \cap A_{k}^{\prime}$. Also it follows that

$$
\begin{aligned}
u^{-j} e_{s, i 1}^{(k+n)} h e_{s, 1 i}^{(k+n)} u^{j}\left(\xi_{1}+\zeta\right) & =u^{-j} e_{s, i 1}^{(k+n)} h u^{j} \alpha^{-j}\left(e_{s, 1 i}^{(k+n)}\right)\left(\xi_{1}+\zeta\right) \\
& \approx u^{-j} e_{s, i 1}^{(k+n)} h\left(\sum c_{s, 1 i ; t, a b}\left(u^{j} e_{t, a b}^{(k+2 n)} \xi_{1}+\zeta(j, t, a, b)\right)=0,\right.
\end{aligned}
$$

where $\alpha^{-j}\left(e_{s, 1 i}^{(k+n)}\right) 1_{k}=\sum c_{s, 1 i ; t, a b} e_{t, a b}^{(k+2 n)} 1_{k}$. Here we have used the fact that $u^{j} e_{t, a b}^{(k+2 n)} \zeta \approx$ $u^{j} e_{t, a b}^{(k+2 n)} \eta_{1} \approx \zeta(j, t, a, b)$. In the same way we obtain that

$$
\begin{aligned}
u^{-j} e_{s, i 1}^{(k+n)} h e_{s, 1 i}^{(k+n)} u^{j}\left(\xi_{1}-\zeta\right) & =u^{-j} e_{s, i 1}^{(k+n)} h u^{j} \alpha^{-j}\left(e_{s, 1 i}^{(k+n)}\right)\left(\xi_{1}-\zeta\right) \\
& \approx u^{-j} e_{s, i 1}^{(k+n)}\left(\sum c_{s, 1 i ; t, a b}\left(u^{j} e_{t, a b}^{(k+2 n)} \xi_{1}-\zeta(j, t, a, b)\right)\right. \\
& \approx u^{-j} e_{s, i 1}^{(k+n)} u^{j} \alpha^{-j}\left(e_{s, 1 i}^{(k+n)}\right)\left(\xi_{1}-\zeta\right) \\
& =\alpha^{-j}\left(e_{s, i i}^{(k+n)}\right)\left(\xi_{1}-\zeta\right) .
\end{aligned}
$$


Then, since $\alpha^{-j}\left(1_{k+n}\right) \geq 1_{k}$, it follows that

$$
\begin{aligned}
& \bar{h}\left(\xi_{1}+\zeta\right) \approx 0 \\
& \bar{h}\left(\xi_{1}-\zeta\right) \approx \xi_{1}-\zeta .
\end{aligned}
$$

Hence

$$
e^{i \pi \bar{h}} \xi_{1}=e^{i \pi \bar{h}}\left(\xi_{1}+\zeta\right) / 2+e^{i \pi \bar{h}}\left(\xi_{1}-\zeta\right) / 2 \approx\left(\xi_{1}+\zeta\right) / 2-\left(\xi_{1}-\zeta\right) / 2 \approx \zeta .
$$

Thus we have that $e^{i \pi \bar{h}} 1_{k} \xi \approx 1_{k} \eta$. Note that $w_{t}=e^{i \pi t \bar{h}}$ in $\mathcal{U}\left(1_{k} B 1_{k}\right)$ almost commutes with $\mathcal{F}$. On the other hand we take a continuous path $\left(v_{t}\right)$ in $\mathcal{U}\left(\left(1-1_{k}\right) B\left(1-1_{k}\right)\right)$ such that $v_{1}\left(1-1_{k}\right) \xi \approx\left(1-1_{k}\right) \eta$. Taking the sum of $w_{t}$ and $v_{t}$ completes the proof in the case $\mathcal{L}_{\xi} \perp \mathcal{L}_{\eta}$.

If $\mathcal{L}_{\xi} \not \perp \mathcal{L}_{\eta}$, then we find a unit vector $\eta^{\prime}$ such that $\mathcal{L}_{\xi} \perp \mathcal{L}_{\eta^{\prime}}, \mathcal{L}_{\eta} \perp \mathcal{L}_{\eta^{\prime}}$, and

$$
\left|\langle x \eta, \eta\rangle-\left\langle x \eta^{\prime}, \eta^{\prime}\right\rangle\right|<\delta^{\prime}, \quad x \in \mathcal{G}
$$

for an arbitrarily small $\delta^{\prime}>0$. We apply the previous argument to the pairs $\xi, \eta^{\prime}$ and $\eta^{\prime}, \eta$ to produce appropriate continuous paths $\left(u_{t}\right),\left(v_{t}\right)$ in $\mathcal{U}(B)$; in particular $u_{1} \xi=\eta^{\prime}$ and $v_{1} \eta^{\prime}=\eta$. Then the product $\left(v_{t} u_{t}\right)$ satisfies the required properties.

To find such an $\eta^{\prime}$ we use the fact that $A \times_{\alpha} \mathbf{Z}$ is not of type I. Note that the set of vector states of $A \times_{\alpha} \mathbf{Z}$ in this representation $\rho=\pi \mid A \times_{\alpha} \mathbf{Z}$ is weak*-dense in the state space of $A \times_{\alpha} \mathbf{Z}$. Hence there is a state $\varphi$ of $A \times_{\alpha} \mathbf{Z}$ such that $\pi_{\varphi}$ is disjoint from $\rho$ and

$$
\left|\omega_{\eta} \rho(x)-\varphi(x)\right|<\delta^{\prime}, \quad x \in \mathcal{G},
$$

where we denote by $\omega_{\eta}$ the state on $\mathcal{B}\left(\mathcal{H}_{\pi}\right)$ defined by the vector $\eta$. Then we can find a sequence $\left(\zeta_{n}\right)$ of unit vectors in $\mathcal{H}_{\pi}$ such that $\omega_{\zeta_{n}} \rho$ converges to $\varphi$ in the weak* topology. Hence $\left(\zeta_{n}\right)$ must converge to zero in the weak topology; we can take the required $\eta^{\prime}$ near $\zeta_{n}$ for a sufficiently large $n$.

From the proof of the above theorem we obtain:

Theorem 4.2 Let $A$ be an $A F C^{*}$-algebra and $\alpha \in \operatorname{Aut}(A)$. Then the crossed product $A \times{ }_{\alpha} \mathbf{Z}$ has the transitivity: For any pair of pure states $\omega_{1}$ and $\omega_{2}$ of $A \times_{\alpha} \mathbf{Z}$ with $\operatorname{ker} \pi_{\omega_{1}}=$ ker $\pi_{\omega_{2}}$ there is an $\beta \in \operatorname{AInn}\left(A \times_{\alpha} \mathbf{Z}\right)$ such that $\omega_{1}=\omega_{2} \beta$.

Proof. If $\omega_{1} \sim \omega_{2}$ then this follows from Kadison's transitivity. Thus we may assume that $\omega_{1}$ and $\omega_{2}$ are not equivalent. Hence at least the quotient $A \times_{\alpha} \mathbf{Z} / \operatorname{ker} \pi_{\omega_{i}}$ does not contain a non-zero type I ideal. We can prove this theorem just as Theorem 2.5 since $A \times{ }_{\alpha} \mathbf{Z}$ has the following property (where $A$ denotes $A \times{ }_{\alpha} \mathbf{Z}$ ).

Property 4.3 For any finite subset $\mathcal{F}$ of $A$ and $\epsilon>0$ there exist a finite subset $\mathcal{G}$ of $A$ and $\delta>0$ satisfying: Let $B$ be $a C^{*}$-algebra such that $B \supset A$. For any pair of pure states 
$\omega_{1}$ and $\omega_{2}$ of $B$ such that $\omega_{1} \sim \omega_{2}, B / \operatorname{ker} \pi_{\omega_{1}}$ does not contain a non-zero type I ideal, and

$$
\left|\omega_{1}(x)-\omega_{2}(x)\right|<\delta, \quad x \in \mathcal{G},
$$

there is a continuous path $\left(u_{t}\right)_{t \in[0,1]}$ in $\mathcal{U}(B)$ such that $u_{0}=1, \omega_{1}=\omega_{2} \operatorname{Ad} u_{1}$, and

$$
\left\|\operatorname{Ad} u_{t}(x)-x\right\|<\epsilon, \quad x \in \mathcal{F} .
$$

Lemma 4.4 Let $A$ be an $A F C^{*}$-algebra and $\alpha \in \operatorname{Aut}(A)$. Then the crossed product $A \times{ }_{\alpha} \mathbf{Z}$ has Property 4.3 .

Proof. The proof of this fact immediately follows from the proof of Theorem 4.1, since the assumption on $B /$ ker $\pi_{\omega_{1}}$ substitutes the condition on $A \times{ }_{\alpha} \mathbf{Z}$ there.

\section{$5 \quad$ Purely infinite $C^{*}$-algebras}

Let $\lambda \in(0,1)$ and let $G_{\lambda}$ be the subgroup of $\mathbf{R}$ generated by $\lambda^{n}$ with $n \in \mathbf{Z}$. Equipping $G_{\lambda}$ with the order coming from $\mathbf{R}, G_{\lambda}$, being dense in $\mathbf{R}$, is a dimension group, i.e., there is a stable AF $C^{*}$-algebra $A_{\lambda}$ whose dimension group is $G_{\lambda} ; A_{\lambda}$ is unique up to isomorphism. Let $m_{\lambda}$ denote the automorphism of $G_{\lambda}$ defined by the multiplication of $\lambda$; there is an automorphism $\alpha_{\lambda}$ of $A_{\lambda}$ which induces $m_{\lambda}$ on $G_{\lambda} ; \alpha_{\lambda}$ is unique up to cocycle conjugacy [7]. If we denote by $\tau$ a (densely-defined lower-semicontinuous) trace on $A_{\lambda}$ (which is unique up to constant multiple), $\alpha_{\lambda}$ satisfies that $\tau \alpha_{\lambda}=\lambda \tau$. Then it follows from [12] that the crossed product $A_{\lambda} \times{ }_{\alpha_{\lambda}} \mathbf{Z}$ is a purely infinite simple $C^{*}$-algebra. We can compute $K_{*}\left(A_{\lambda} \times_{\alpha_{\lambda}} \mathbf{Z}\right)$ by using the Pimsner-Voiculescu exact sequence; $K_{0}=G_{\lambda} /(1-\lambda) G_{\lambda}$ and $K_{1}=0$. When $\{f \in \mathbf{Z}[t] \mid f(\lambda)=0\}=p(t) \mathbf{Z}[t]$ with some $p(t) \in \mathbf{Z}[t]$, we have that $K_{0}=\mathbf{Z} / p(1) \mathbf{Z}$. By using the classification theory [8], we know that we get all the stable Cuntz algebras $\mathcal{O}_{n} \otimes \mathcal{K}$ in this way. Recall that $K_{0}\left(\mathcal{O}_{n}\right)=\mathbf{Z} /(n-1) \mathbf{Z}$ if $n<\infty$ and $K_{0}\left(\mathcal{O}_{\infty}\right)=\mathbf{Z}$. Hence by using 2.11 and 3.5 we have:

Lemma 5.1 All the $C^{*}$-algebras of the form

$$
C(\mathbf{T}) \otimes M_{k} \otimes \mathcal{O}_{n}
$$

have Property 2.9, where $n=2,3, \ldots, \infty$ with $n=\infty$ inclusive and $k=1,2, \ldots, n-1$ if $n<\infty$ and $k=1,2, \ldots$ otherwise. Moreover all the $C^{*}$-algebras obtained as inductive limits of finite direct sums of $C^{*}$-algebras of the above form have Property 2.9.

Note that $K_{0}\left(C(\mathbf{T}) \otimes M_{k} \otimes \mathcal{O}_{n}\right)=K_{0}\left(\mathcal{O}_{n}\right)$ and $K_{1}\left(C(\mathbf{T}) \otimes M_{k} \otimes \mathcal{O}_{n}\right)=K_{0}\left(\mathcal{O}_{n}\right)$. Tensoring with $M_{k}$ exhausts all possible pairs $\left(K_{0},[1]\right)$ for cyclic groups $K_{0}$. In [2] a class of inductive limit $C^{*}$-algebras is considered using the $C^{*}$-algebras in the above lemma as building blocks. It is not difficult to show that the purely infinite simple separable unital 
$C^{*}$-algebras obtained this way exhaust all possible $\left(G_{0}, g, G_{1}\right)$ for $\left(K_{0},[1], K_{1}\right)$, where $G_{0}$ and $G_{1}$ are arbitrary countable abelian groups and $g \in G_{0}$. (Given any pair of countable abelian groups $G_{0}, G_{1}$ and $g \in G_{0}$ we find an inductive system $\left(G_{i n}, \phi_{i n}\right)$ whose limit is $G_{i}$, where all $G_{i n}$ is a finite direct sum of cyclic groups; for $i=0$ we specify $g_{0 n} \in G_{0 n}$ with $\phi_{0 n}\left(g_{0 n}\right)=g_{0, n+1}$ so that $\left(g_{0 n}\right)$ represents $g$. Let $G_{n}=G_{0 n} \oplus G_{1 n}$ and $g_{n}=g_{0 n} \oplus 0$ and extend $\phi_{i n}: G_{i n} \rightarrow G_{i, n+1}$ to a map $\phi_{i n}: G_{n} \rightarrow G_{n+1}$ by adding zero maps. Then it follows that $G_{i}$ is the inductive limit of $\left(G_{n}, \phi_{i n}\right)$ for $i=0,1$. For each $G_{n}$ we take a direct sum $A_{n}$ of $C^{*}$-algebras of the form $C(\mathbf{T}) \otimes M_{k} \otimes \mathcal{O}_{n}$ with $K_{*}\left(A_{n}\right)=G_{n}$ and [1] $=g_{n}$. Then we find a unital homomorphism $\varphi_{n}: A_{n} \rightarrow A_{n+1}$ such that $\varphi_{n}$ induces $\phi_{i n}$ for $i=0,1$ and $\varphi_{n}$ is a non-zero map from each direct summand of $A_{n}$ into each direct summand of $A_{n+1}$; moreover $\varphi_{n}$ maps the canonical unitary $z \otimes 1 \otimes 1$ of each direct summand $C(\mathbf{T}) \otimes M_{k} \otimes \mathcal{O}_{n}$ of $A_{n}$ to a non-zero unitary $u$ for each direct summand of $A_{n+1}$ such that the spectrum of $u$ evaluated at each $t \in \mathbf{T}$ is $\mathbf{T}$ (a considerably weaker condition will suffice; see [2] for details); the latter is imposed to insure that the inductive limit $A$ of $\left(A_{n}, \varphi_{n}\right)$ is simple. Then $A$ satisfies that $K_{i}(A)=G_{i}$ and $[1]=g$.)

Let $\mathcal{C}_{\infty}$ denote the class of purely infinite separable simple $C^{*}$-algebras satisfying the Universal Coefficient Theorem, i.e., $\mathcal{C}_{\infty}$ is the class of $C^{*}$-algebras classified in terms of $\mathrm{K}$ theory by Kirchberg and Phillips [8, 10]. Since all of $\mathcal{C}_{\infty}$ can be obtained as inductive limits as discussed above, we have:

Theorem 5.2 Any $C^{*}$-algebra $A$ in $\mathcal{C}_{\infty}$ has Property 2.9. In particular $\operatorname{AInn}(A)$ acts transitively on $P(A)$.

\section{The group $C^{*}$-algebras of discrete amenable groups}

Theorem 6.1 If $G$ is a countable discrete amenable group, then the group $C^{*}$-algebra $C^{*}(G)$ satisfies the transitivity: for any pair of pure states $\omega_{1}$ and $\omega_{2}$ with $\operatorname{ker} \pi_{\omega_{1}}=\operatorname{ker} \pi_{\omega_{2}}$ there is an $\alpha \in \operatorname{AInn}\left(C^{*}(G)\right)$ such that $\omega_{1}=\omega_{2} \alpha$.

Proof. If $\omega_{1} \sim \omega_{2}$ then this follows from Kadison's transitivity. Thus we may assume that $\omega_{1}$ and $\omega_{2}$ are not equivalent. Hence at least the quotient $C^{*}(G) / I$ with $I=\operatorname{ker} \pi_{\omega_{i}}$ does not contain a non-zero type I ideal. We can prove this theorem just as Theorem 2.5 (or 4.2) once we have shown the following:

Lemma 6.2 If $G$ is a discrete amenable group, $C^{*}(G)$ has Property 4.3.

Proof. Since $\mathrm{G}$ is a discrete amenable group, for any finite subset $\mathcal{F}$ of $G$ and $\epsilon>0$ there is a finite subset $\mathcal{G}$ of $G$ such that

$$
\frac{|\mathcal{G} \Delta \mathcal{G} g|}{|\mathcal{G}|}<\epsilon, \quad g \in \mathcal{F},
$$


where $\Delta$ denotes the difference of sets and $|\cdot|$ denotes the number of elements. We may suppose that $\mathcal{G} \ni 1$.

Suppose that $C^{*}(G)$ is a unital $C^{*}$-subalgebra of $B$ and let $\pi$ be an irreducible representation of $B$. Let $\delta>0$ and let $\xi, \eta$ be unit vectors in $\mathcal{H}_{\pi}$ such that

$$
|\langle\pi(g) \xi, \pi(h) \xi\rangle-\langle\pi(g) \eta, \pi(h) \eta\rangle|<\delta
$$

for $g, h \in \mathcal{G}$.

First suppose that the linear subspace $\mathcal{L}_{\xi}$ spanned by $\pi(g) \xi, g \in \mathcal{G}$ and the linear subspace $\mathcal{L}_{\eta}$ spanned by $\pi(g) \eta, g \in \mathcal{G}$ are mutually orthogonal. Then for a sufficiently small $\delta>0$ there is a family $(\zeta(g))$ of vectors in $\mathcal{H} \ominus \mathcal{L}_{\xi}$, which is infinite-dimensional, such that

$$
\|\pi(g) \eta-\zeta(g)\|<\epsilon^{\prime}, \quad g \in \mathcal{G}
$$

for small $\epsilon^{\prime}>0$ and

$$
\langle\pi(g) \xi, \pi(h) \xi\rangle=\langle\zeta(g), \zeta(h)\rangle .
$$

Since $\pi(g) \zeta \approx \pi(g) \eta \approx \zeta(g)$ for $g \in \mathcal{G}$ with $\zeta=\zeta(1)$, we have that $\|\pi(g) \zeta-\zeta(g)\|<2 \epsilon^{\prime}$. Then there is a projection $E$ on $\mathcal{L}_{\xi}+\mathcal{L}_{\zeta}$ such that

$$
\begin{aligned}
& E(\pi(g) \xi+\zeta(g))=0 \\
& E(\pi(g) \xi-\zeta(g))=\pi(g) \xi-\zeta(g)
\end{aligned}
$$

where $\mathcal{L}_{\zeta}$ is the subspace spanned by $\zeta(g), g \in \mathcal{G}$. Then we find an $h=h^{*} \in B$ such that $\|h\|=1$ and

$$
\pi(h) \mid\left(\mathcal{L}_{\xi}+\mathcal{L}_{\zeta}\right)=E
$$

Set

$$
\bar{h}=\frac{1}{|\mathcal{G}|} \sum_{g \in \mathcal{G}} g^{-1} h g,
$$

which is a self-adjoint element in $B$ with norm at most one and satisfies that $\left\|g^{-1} \bar{h} g-\bar{h}\right\| \leq$ $\epsilon, g \in \mathcal{F}$. Note that for $g \in \mathcal{G}$,

$$
\left\|\pi\left(g^{-1} h g\right)(\xi+\zeta)\right\|<2 \epsilon^{\prime}
$$

and

$$
\left\|\pi\left(g^{-1} h g\right)(\xi-\zeta)-(\xi-\zeta)\right\|<2 \epsilon^{\prime} .
$$

Then we obtain that $\pi\left(e^{i \pi \bar{h}}\right) \xi \approx \zeta$ because

$$
\begin{aligned}
& \left\|\pi\left(e^{i \pi \bar{h}}\right) \xi-\zeta\right\| \\
& \leq\left\|\sum_{m=1}^{\infty} \frac{(i \pi)^{m}}{m !} \pi\left(\bar{h}^{m}\right) \frac{1}{2}(\xi+\zeta)\right\|+\left\|\sum_{m=1}^{\infty} \frac{(i \pi)^{m}}{m !}\left(\pi\left(\bar{h}^{m}\right)-1\right) \frac{1}{2}(\xi-\zeta)\right\| \\
& \leq \epsilon^{\prime}\left(e^{\pi}-1+\pi e^{\pi}\right) .
\end{aligned}
$$


Define $u_{t}=e^{i t \pi \bar{h}}$, which is a continuous path in $\mathcal{U}(B)$ satisfying $\left\|\left[u_{t}, g\right]\right\|<\pi \epsilon, g \in \mathcal{F}$ and $\pi\left(u_{1}\right) \xi \approx \zeta \approx \eta$. Hence this completes the proof in the case that $\mathcal{L}_{\xi} \perp \mathcal{L}_{\eta}$.

If $\mathcal{L}_{\xi} \not \perp \mathcal{L}_{\eta}$, then we first find a unit vector $\eta^{\prime}$ such that $\mathcal{L}_{\xi} \perp \mathcal{L}_{\eta^{\prime}}, \mathcal{L}_{\eta} \perp \mathcal{L}_{\eta^{\prime}}$, and

$$
\left|\langle\pi(g) \eta, \pi(h) \eta\rangle-\left\langle\pi(g) \eta^{\prime}, \pi(h) \eta^{\prime}\right\rangle\right|<\delta^{\prime}, \quad g, h \in \mathcal{G}
$$

for a very small $\delta^{\prime}>0$. We apply the previous argument to the pairs $\xi, \eta^{\prime}$ and $\eta^{\prime}, \eta$ to get the conclusion.

To find such an $\eta^{\prime}$ we may argue as in the proof of 2.3 (see also the final part of the proof of 4.1). Let $e \in B$ be such that $0 \leq e \leq 1$ and

$$
\left\|e h^{-1} g e-\omega_{2}\left(\pi\left(h^{-1} g\right)\right) e^{2}\right\|<\delta^{\prime}, \quad g, h \in \mathcal{G},
$$

where $\omega_{2}$ is the vector state defined by $\eta$. If there is no such $\eta^{\prime}$, then the spectral projection of $\pi(e)$ corresponding to $\left[1-\delta^{\prime}, 1\right]$ must be finite-dimensional; i.e., $\pi(B)$ contains the compact operators on $\mathcal{H}_{\pi}$, which is excluded by the assumption.

\section{$7 \quad$ Strong transitivity}

We first introduce the following property for a $C^{*}$-algebra $A$ which is stronger than 2.9:

Property 7.1 For any finite subset $\mathcal{F}$ of $A$ and $\epsilon>0$ there exist a finite subset $\mathcal{G}$ of $A$ and $\delta>0$ satisfying: If $B$ is a $C^{*}$-algebra containing $A$ as a $C^{*}$-subalgebra and $\omega_{1}, \omega_{2}, \ldots, \omega_{n}, \varphi_{1}, \varphi_{2}, \ldots, \varphi_{n}$ are pure states of $B$ such that

1. $\left(\omega_{i}\right)$ are mutually disjoint,

2. $\omega_{i} \sim \varphi_{i}$ for $i=1,2, \ldots, n$,

3. $\left|\omega_{i}(x)-\varphi_{i}(x)\right|<\delta, \quad x \in \mathcal{G}, i=1,2, \ldots, n$,

then there is a continuous path $\left(u_{t}\right)_{t \in[0,1]}$ in $\mathcal{U}(B)$ such that $u_{0}=1, \omega_{i}=\varphi_{i} \operatorname{Ad} u_{1}, \quad i=$ $1,2, \ldots, n$, and

$$
\left\|\operatorname{Ad} u_{t}(x)-x\right\|<\epsilon, \quad x \in \mathcal{F}, t \in[0,1]
$$

We can actually show the above property for all the $C^{*}$-algebras for which we have shown Property 2.9. This is because we can use the same argument for each pair $\omega_{i}, \varphi_{i}$ up to the point where we invoke Kadison's transitivity; instead of working in one irreducible representation of $B$ we are now working in $\bigoplus_{i=1}^{n} \pi_{\omega_{i}}$, which is a direct sum of mutually disjoint irreducible representations. We have to find an element $h$ in the $C^{*}$-algebra $B$ with the prescribed property in this larger space. But there is a form of Kadison's transitivity in this generality (cf. 1.21.16 of [13]); so we are done.

The following follows just like 2.3. 
Lemma 7.2 If $\omega_{1}, \omega_{2}, \ldots, \omega_{n}, \varphi_{1}, \varphi_{2}, \ldots, \varphi_{n}$ are pure states of $A$ such that

1. $\left(\varphi_{i}\right)$ are mutually disjoint,

2. $\operatorname{ker} \pi_{\omega_{i}}=\operatorname{ker} \pi_{\varphi_{i}}$ for $i=1,2, \ldots, n$,

then for any finite subset $\mathcal{F}$ of $A$ and $\epsilon>0$ there is a $u \in \mathcal{U}(A)$ such that

$$
\left|\omega_{i}(x)-\varphi_{i} \operatorname{Ad} u(x)\right|<\epsilon, \quad x \in \mathcal{F}, i=1,2, \ldots, n .
$$

Theorem 7.3 Suppose that $A$ is a separable $C^{*}$-algebra satisfying Property 7.1. Let $\left(\omega_{i}\right)_{1 \leq i \leq n}$ and $\left(\varphi_{i}\right)_{1 \leq i \leq n}$ be finite sequences of pure states of $A$ such that $\left(\omega_{i}\right)\left(\right.$ resp. $\left.\left(\varphi_{i}\right)\right)$ are mutually disjoint and $\operatorname{ker} \pi_{\omega_{i}}=\operatorname{ker} \pi_{\varphi_{i}}$ for all $i$. Then there is an $\alpha \in \operatorname{AInn}(A)$ such that $\omega_{i}=\varphi_{i} \alpha$ for all $i=1,2, \ldots, n$. In particular if $\left(\omega_{i}\right)_{1 \leq i \leq n+1}$ are pure states such that they are mutually disjoint maybe except for the pair $\omega_{1}, \omega_{n+1}$ and all ker $\pi_{\omega_{i}}$ 's are equal, then there is an $\alpha \in \operatorname{AInn}(A)$ such that $\omega_{i} \alpha=\omega_{i+1}, i=1,2, \ldots, n$.

The proof goes just like the proof of 2.5 does. We present what we use at each induction step in a form of lemma:

Lemma 7.4 Suppose that $A$ satisfies 7.1. For any finite subset $\mathcal{F}$ of $A$ and $\epsilon>0$ there exist a finite subset $\mathcal{G}$ of $A$ and $\delta>0$ satisfying: If $\omega_{i}, \varphi_{i}, i=1,2, \ldots, n$, are pure states of $A$ such that

1. $\left(\varphi_{i}\right)$ are mutually disjoint,

2. $\operatorname{ker} \pi_{\omega_{i}}=\operatorname{ker} \pi_{\varphi_{i}}$ for all $i$,

3. $\left|\omega_{i}(x)-\varphi_{i}(x)\right|<\delta, \quad x \in \mathcal{G}, i=1,2, \ldots, n$,

then for any finite subset $\mathcal{F}^{\prime}$ of $A$ and $\epsilon^{\prime}>0$ there is a continuous path $\left(u_{t}\right)_{t \in[0,1]}$ in $\mathcal{U}(A)$ such that $u_{0}=1$,

$$
\begin{aligned}
\left|\omega_{i}(x)-\varphi_{i} \operatorname{Ad} u_{1}(x)\right| & <\epsilon^{\prime}, x \in \mathcal{F}^{\prime}, i=1,2, \ldots, n, \\
\left\|\operatorname{Ad} u_{t}(x)-x\right\| & <\epsilon, \quad x \in \mathcal{F}, t \in[0,1] .
\end{aligned}
$$

Finally we present another version of transitivity:

Theorem 7.5 Suppose that $A$ is a separable $C^{*}$-algebra with Property 7.1. Let $\left(\pi_{n}\right)$ and $\left(\rho_{n}\right)$ be sequences of irreducible representations of $A$ such that $\left(\pi_{n}\right)\left(\right.$ resp. $\left.\left(\rho_{n}\right)\right)$ are mutually disjoint and $\operatorname{ker} \pi_{n}=\operatorname{ker} \rho_{n}$ for all $n$. Then there is an $\alpha \in \operatorname{AInn}(A)$ such that $\pi_{n}=\rho_{n} \alpha$ for all $n \in \mathbf{N}$. In particular if $\left(\pi_{n}\right)_{n \in \mathbf{Z}}$ are irreducible representations of $A$ such that they are mutually disjoint and all $\operatorname{ker} \pi_{n}$ 's are equal, then there is an $\alpha \in \operatorname{AInn}(A)$ such that $\pi_{n} \alpha=\pi_{n+1}$ for all $n$. 
Proof. The proof is similar to the one of 7.3 . We will construct pure states $\omega_{n}$ (resp. $\varphi_{n}$ ) associated with $\pi_{n}$ (resp. $\rho_{n}$ ) inductively such that $\omega_{n}=\varphi_{n} \alpha$ holds for all $n$. To introduce a new pair of pure states at each induction step we will use the following easy lemma.

Lemma 7.6 Let $\pi$ and $\rho$ be mutually disjoint irreducible representations of $A$ with ker $\pi=$ ker $\rho$ and $u \in \mathcal{U}(A)$. Then for any finite subset $\mathcal{G}$ of $A$ and $\delta>0$ there are a pure state $\omega$ associated with $\pi$ and a pure state $\varphi$ associated with $\rho$ such that $|\omega(x)-\varphi \operatorname{Ad} u(x)|<$ $\delta, x \in \mathcal{G}$.

Remark 7.7 If $A$ is a non type I separable simple $C^{*}$-algebra with Property 7.1 , it follows from the above theorem that there is an $\alpha \in \operatorname{AInn}(A)$ such that all non-zero powers $\alpha^{n}$ are outer. Since for any injective map $\omega$ of $\mathbf{Z}$ into the set of equivalence classes of irreducible representations of $A$ there is an $\alpha \in \operatorname{AInn}(A)$ such that $\omega(n) \alpha=\omega(n+1), n \in \mathbf{Z}$, it follows that the quotient $\operatorname{AInn}(A) / \operatorname{Inn}(A)$ is uncountable.

\section{Remarks on Property 2.9}

Looking at the proofs of 3.43 .8 and 4.1 etc., we come to know that the path $\left(u_{t}\right)$ in Property 2.9 may be chosen so that its length is dominated by a universal constant, which is only slightly bigger than $\pi$. (This follows by modifying the proofs given there; it is $2 \pi$ instead of $\pi$ that follows immediately.) Taking this fact into consideration, we first introduce the following stronger condition:

Property 8.1 (for a $C^{*}$-algebra $A$ and a constant $C \geq \pi$ ) For any finite subset $\mathcal{F}$ of $A$ and $\epsilon>0$ there exist a finite subset $\mathcal{G}$ of $A$ and $\delta>0$ satisfying: If $B$ is a $C^{*}$-algebra containing $A$ as a $C^{*}$-subalgebra and $\omega_{1}$ and $\omega_{2}$ are pure states of $B$ such that $\omega_{1} \sim \omega_{2}$ and

$$
\left|\omega_{1}(x)-\omega_{2}(x)\right|<\delta, \quad x \in \mathcal{G},
$$

then for any $\epsilon^{\prime}>0$ there is a rectifiable path $\left(u_{t}\right)_{t \in[0,1]}$ in $\mathcal{U}(B)$ such that $u_{0}=1, \omega_{1}=$ $\omega_{2} \operatorname{Ad} u_{1}$, length $\left(\left(u_{t}\right)\right)<C+\epsilon^{\prime}$, and

$$
\left\|\operatorname{Ad} u_{t}(x)-x\right\|<\epsilon, \quad x \in \mathcal{F}, t \in[0,1]
$$

As asserted above, in the cases we handled in the previous sections, the $C^{*}$-algebra $A$ has this property for $C=\pi$. If $A=\mathbf{C}$, then $C=\pi / 2$ suffices for the above property to hold. If the property holds for all $A=\mathbf{C}^{n}$ with $n \in \mathbf{N}$ and for a constant $C$, then one can check that $C \geq \pi$. To illustrate these points we give two propositions:

Proposition 8.2 Let $\mathcal{H}$ be a Hilbert space and $e$ be a projection in $\mathcal{H}$. If $\xi$ and $\eta$ are unit vectors in $\mathcal{H}$ such that $\langle e \xi, \xi\rangle=\langle e \eta, \eta\rangle$, there is a rectifiable path $\left(u_{t}\right)_{t \in[0,1]}$ in the group $\mathcal{U}(\mathcal{H})$ of unitaries on $\mathcal{H}$ such that $u_{0}=1, u_{1} \xi=\lambda \eta$ for some $\lambda \in \mathbf{T},\left[u_{t}, e\right]=0$, and length $\left(\left(u_{t}\right)\right) \leq \pi / 2$. 
Proof. There is a $\lambda \in \mathbf{T}$ such that

$$
\Re\langle e \xi, \lambda \eta\rangle \geq 0, \quad \Re\langle(1-e) \xi, \lambda \eta\rangle \geq 0 .
$$

Hence we only have to apply the following proposition to the pairs $e \xi, \lambda e \eta \in e \mathcal{H}$ and $(1-e) \xi, \lambda(1-e) \eta \in(1-e) \mathcal{H}$ separately to reach the conclusion.

Proposition 8.3 Let $\mathcal{H}$ be a Hilbert space and $\xi, \eta$ unit vectors in $\mathcal{H}$. If $\theta \in[0, \pi]$ is such that $\Re\langle\xi, \eta\rangle=\cos \theta$, then there is a rectifiable path $\left(u_{t}\right)$ in $\mathcal{U}(\mathcal{H})$ such that $u_{0}=1$, $u_{1} \xi=\eta$, and length $\left(\left(u_{t}\right)\right)=\theta$. Moreover for any rectifiable path $\left(v_{t}\right)$ in $\mathcal{U}(\mathcal{H})$ with $v_{0}=1$ and $v_{1} \xi=\eta$, it follows that length $\left(\left(v_{t}\right)\right) \geq \theta$.

Proof. If $\eta=e^{ \pm i \theta} \xi$, then we set $u_{t}=e^{ \pm i t \theta}$.

Suppose that $\xi$ and $\eta$ are linearly independent. For $t \in[0,1]$ let

$$
\xi(t)=\cos \theta t \cdot \xi+\sin \theta t(\sin \theta)^{-1}(\eta-\cos \theta \cdot \xi) .
$$

Then $\xi(0)=\xi$ and $\xi(1)=\eta$. Since $\Re\langle\xi, \eta-\cos \theta \cdot \xi\rangle=0$ and $\|\eta-\cos \theta \cdot \xi\|=\sin \theta$, it follows that $\|\xi(t)\|=1$ and $\left\|\xi^{\prime}(t)\right\|=\theta$. Thus $(\xi(t))$ is a $C^{1}$ path in the unit vectors of $\mathcal{H}$ and its length is $\theta$.

We define $\alpha(t) \in \mathbf{R}$ by

$$
i \alpha(t)=\left\langle\xi^{\prime}(t), \xi(t)\right\rangle=i \theta(\sin \theta)^{-1} \Im\langle\eta, \xi\rangle
$$

and $\beta(t) \in \mathbf{R}$ by

$$
\beta(t)=\left\|\xi^{\prime}(t)-i \alpha(t) \xi(t)\right\|=(\theta-\alpha(t))^{1 / 2} .
$$

Since both $\alpha(t)$ and $\beta(t)$ are constants, we write $\alpha(t)=\alpha$ and $\beta(t)=\beta$. Let $\zeta(t)=$ $\beta^{-1}\left(\xi^{\prime}(t)-i \alpha \xi(t)\right)$ and define a self-adjoint operator $h(t)$ by

$$
h(t)=\alpha \xi(t) \otimes \xi(t)-i \beta \zeta(t) \otimes \xi(t)+i \beta \xi(t) \otimes \zeta(t)-\alpha \zeta(t) \otimes \zeta(t) .
$$

Then $(h(t))$ is a continuous path in the set of self-adjoint operators in $\mathcal{H}$ such that $i h(t) \xi(t)=\xi^{\prime}(t)$ and

$$
\|h(t)\|=\left(\alpha^{2}+\beta^{2}\right)^{1 / 2}=\theta=\left\|\xi^{\prime}(t)\right\| .
$$

We define a $C^{1}$ path $(u(t))_{t \in[0,1]}$ in $\mathcal{U}(\mathcal{H})$ by

$$
\frac{d}{d t} u(t)=i h(t) u(t), \quad u(0)=1 .
$$

In fact, since $\frac{d}{d t} u(t)^{*} u(t)=0=\frac{d}{d t} u(t) u(t)^{*}$, we have that $u(t)^{*} u(t)=1=u(t) u(t)^{*}$. We also have that length $((u(t))=\theta$. Since $u(0) \xi=\xi=\xi(0)$ and

$$
\begin{aligned}
\frac{d}{d t}\|u(t) \xi-\xi(t)\|^{2} & =\frac{d}{d t}(2-2 \Re\langle u(t) \xi, \xi(t)\rangle) \\
& =-2 \Re\langle i h(t) u(t) \xi, \xi(t)\rangle-2 \Re\left\langle u(t) \xi, \xi^{\prime}(t)\right\rangle \\
& =0,
\end{aligned}
$$


we have that $u(t) \xi=\xi(t)$; in particular $u(1) \xi=\eta$. Thus $(u(t))$ is the desired path in $\mathcal{U}(\mathcal{H})$.

Let $\left(v_{t}\right)$ be a rectifiable path in $\mathcal{U}(\mathcal{H})$ such that $v_{0}=1$ and $v_{1} \xi=\eta$. Since

$$
2 \cos \theta=2 \Re\langle\xi, \eta\rangle=\left\langle\left(v_{1}^{*}+v_{1}\right) \xi, \xi\right\rangle,
$$

it follows that the spectrum $\operatorname{Spec}\left(v_{1}\right)$ has an $e^{i \varphi} \operatorname{such}$ that $\cos \varphi \leq \cos \theta=\Re\langle\xi, \eta\rangle$. For any $\epsilon>0$ we find a sequence $\left(t_{0}, t_{1}, \ldots, t_{n}\right)$ in $[0,1]$ such that $t_{0}=0<t_{1}<t_{2}<\cdots<t_{n}=1$ and $\left\|v_{t_{i}}-v_{t_{i-1}}\right\|<\epsilon$. Then, by the following lemma, we find a $\lambda_{i} \in \operatorname{Spec}\left(v_{t_{i}}\right)$ such that $\lambda_{n}=e^{i \varphi}$ and $\left|\lambda_{i}-\lambda_{i-1}\right| \leq\left\|v_{t_{i}}-v_{t_{i-1}}\right\|$. Hence

$$
\sum_{i=1}^{n}\left|\lambda_{i}-\lambda_{i-1}\right| \leq \sum_{i=0}^{n}\left\|v_{t_{i}}-v_{t_{i-1}}\right\| \leq \operatorname{length}\left(\left(v_{t}\right)\right) \text {. }
$$

Since $\lambda_{0}=1, \lambda_{n}=e^{i \varphi}$ and $\epsilon>0$ is arbitrary, we obtain that $\varphi \leq \operatorname{length}\left(\left(v_{t}\right)\right)$. Since $\theta \leq \varphi$, this completes the proof.

Lemma 8.4 Let $A$ be a $C^{*}$-algebra and let $u, v \in \mathcal{U}(A)$. If $\lambda \in \operatorname{Spec}(u)$, then there is a $\mu \in \operatorname{Spec}(v)$ such that $|\lambda-\mu| \leq\|u-v\|$.

Proof. Let $\delta=\|u-v\|$ and $w=u^{*} v$. Since $\|1-w\|=\delta$, it follows that $\operatorname{Spec}(w) \subset$ $\left\{e^{i t}|| t \mid \leq \theta\right\}=\mathbf{T}_{\theta}$, where $\theta=2 \sin ^{-1} \delta / 2$. Let $\omega$ be a state of $A$ such that $\omega(u)=$ $\lambda \in \operatorname{Spec}(u)$. Then $\omega(w)=\omega\left(u^{*} v\right)=\bar{\lambda} \omega(v)$. Hence $\lambda \omega(w)=\omega(v)$ belongs to the convex closure of $\lambda \mathbf{T}_{\theta}$, which implies that $\operatorname{Spec}(v) \cap \lambda \mathbf{T}_{\theta} \neq \emptyset$.

We seem to need the above stronger property to prove:

Proposition 8.5 If a $C^{*}$-algebra $A$ has Property 8.1 for a constant $C \geq \pi$ and $A_{1}$ is a hereditary $C^{*}$-subalgebra of $A$, then $A_{1}$ has Property 8.1 for the same constant $C$.

To show this we first present the non-unital version of 2.10:

Lemma 8.6 When $A$ is non-unital, Property 8.1 is equivalent to the one obtained by restricting the ambient $C^{*}$-algebra $B$ to a $C^{*}$-algebra having an approximate identity for $A$ as an approximate identity for $B$ itself.

Proof. Technically the proof will be similar to the proofs of 3.7 and 3.8 .

We assume the weaker property for $A$ : For $(\mathcal{F}, \epsilon)$ there is a $(\mathcal{G}, \delta)$ satisfying: If $B \supset A$ and $B=\overline{A B A}$, and $\omega_{1}$ and $\omega_{2}$ are pure states of $B$ such that $\omega_{1} \sim \omega_{2}$ and $\left|\omega_{1}(x)-\omega_{2}(x)\right|<$ $\delta, x \in \mathcal{G}$, then for any $\epsilon^{\prime}>0$ there is a rectifiable path $\left(u_{t}\right)_{t \in[0,1]}$ in $\mathcal{U}(B)$ such that $u_{0}=1$, $\omega_{1}=\omega_{2} \operatorname{Ad} u_{1}$, length $\left(\left(u_{t}\right)\right)<C+\epsilon^{\prime}$, and $\left\|\operatorname{Ad} u_{t}(x)-x\right\|<\epsilon, x \in \mathcal{F}, t \in[0,1]$.

Let $\mathcal{F}$ be a finite subset of $A$ and $\epsilon>0$. We may assume that $\|x\| \leq 1$ for $x \in \mathcal{F}, \epsilon>0$ is sufficiently small, and that there is an $e \in A$ such that $0 \leq e \leq 1$ and $e x e=x, x \in \mathcal{F}$. Let $B$ be a $C^{*}$-algebra with $B \supset A$. 
Let $\epsilon^{\prime}>0$ be such that $2 \sqrt{\epsilon^{\prime}}\left(C+8 \sqrt{\epsilon^{\prime}}\right)<\epsilon$ and let $M, N \in \mathbf{N}$ be so large that $M \epsilon^{\prime 2}>4$ and $N \epsilon^{\prime}>2 C$. There is a sequence $\left(e_{0}, e_{1}, \ldots, e_{M(N+1)}\right)$ in $A$ such that $e_{0} x e_{0}=x, x \in \mathcal{F}, 0 \leq e_{i} \leq 1$, and $e_{i} e_{i-1}=e_{i-1}$. Let $d>1$ be a very large constant and let $\mathcal{F}_{1}=\mathcal{F} \cup\left\{d e_{i} \mid 0 \leq i \leq M(N+1)\right\}$. For $\left(\mathcal{F}_{1}, \epsilon\right)$ we choose a $(\mathcal{G}, \delta)$ as in the weaker version of Property 8.1. We may assume that $\|x\| \leq 1, x \in \mathcal{G}$ and $\delta<1$.

Let $\pi$ be an irreducible representation of $B$ and $\xi, \eta$ unit vectors in $\mathcal{H}_{\pi}$. Suppose that

$$
|\langle\pi(x) \xi, \xi\rangle-\langle\pi(x) \eta, \eta\rangle|<\delta \epsilon^{\prime 2} / 2, \quad x \in \mathcal{G} .
$$

There exists a $j$ between 1 and $(M-1)(N+1)$ inclusive such that

$$
\left\langle\pi\left(e_{j+N}-e_{j-1}\right) \xi, \xi\right\rangle+\left\langle\pi\left(e_{j+N}-e_{j-1}\right) \eta, \eta\right\rangle<\epsilon^{\prime 2} / 2 .
$$

(Otherwise we would be led to a contradiction, $M \epsilon^{\prime 2} / 2 \leq 2$.) If $\left\langle\pi\left(e_{j-1}\right) \xi, \xi\right\rangle>\epsilon^{\prime 2}$, then we have that for $\xi_{1}=\left\|\pi\left(e_{j-1}^{1 / 2}\right) \xi\right\|^{-1} \pi\left(e_{j-1}^{1 / 2}\right) \xi$ and $\eta_{1}=\left\|\pi\left(e_{j-1}^{1 / 2}\right) \eta\right\|^{-1} \pi\left(e_{j-1}^{1 / 2}\right) \eta$,

$$
\left|\left\langle\pi(x) \xi_{1}, \xi_{1}\right\rangle-\left\langle\pi(x) \eta_{1}, \eta_{1}\right\rangle\right|<\delta, \quad x \in \mathcal{G} .
$$

Here we have used that

$$
\left|1-\frac{\left\langle\pi\left(e_{j-1}\right) \eta, \eta\right\rangle}{\left\langle\pi\left(e_{j-1}\right) \xi, \xi\right\rangle}\right|<\delta / 2 .
$$

Then, since $\xi_{1}, \eta_{1} \in \pi(A) \mathcal{H}_{\pi}$, there is a rectifiable path $\left(u_{t}\right)$ in $\mathcal{U}(\overline{A B A})$ such that $u_{0}=1$, $\pi\left(u_{1}\right) \xi_{1}=\eta_{1}$, length $\left(\left(u_{t}\right)\right)<C+\epsilon^{\prime}$, and $\left\|\operatorname{Ad} u_{t}(x)-x\right\|<\epsilon, x \in \mathcal{F}_{1}$. Moreover we may assume that the length of $\left(u_{t}\right)_{t \in[0, s]}$ is proportional to $s$ for any $s \in[0,1]$. We set $p_{k}=e_{j+k}-e_{j+k-1}$ and define

$$
z_{t}=u_{t} e_{j}+\sum_{k=1}^{N-1} u_{(1-k / N) t} p_{k}+1-e_{j+N-1} .
$$

Let $B_{0}$ be the closure of $e_{j+N-1} B e_{j+N-1}$. Then $\left(z_{t}\right)$ is a path in $B_{0}+1$, from which we shall construct a path $\left(v_{t}\right)$ in $\mathcal{U}\left(B_{0}\right)$ with appropriate properties.

Note that $\left\|\left[u_{t}, e_{i}\right]\right\|<\epsilon / d$, where $d \gg 1$ is chosen independently of $N$; i.e., $\left\|\left[u_{t}, e_{i}\right]\right\| \approx$ 0 . Since $z_{t} x=u_{t} x$ and $x z_{t} \approx x u_{t}$ for $x \in \mathcal{F}$, we have that $\left\|\left[z_{t}, x\right]\right\| \approx\left\|\left[u_{t}, x\right]\right\|<\epsilon$ for $x \in \mathcal{F}$. Thus by making $d$ sufficiently large, we may assume that $\left\|\left[z_{t}, x\right]\right\|<\epsilon, x \in \mathcal{F}$.

Let $p_{0}=e_{j}$ and $p_{N}=1-e_{j+N-1}$. Then $\sum_{k=0}^{N} p_{k}=1$ and $p_{k} p_{\ell}=0$ if $|k-\ell|>1$. Let $s\left(p_{k}\right)$ denote the support projection of $p_{k}$ in $B^{* *}$. Then we have that

$$
\begin{aligned}
& \left\|\left(z_{t}-u_{(1-k / N) t}\right) s\left(p_{k}\right)\right\|^{2} \\
& =\left\|\left(u_{(1-(k-1) / N) t} p_{k-1}+u_{(1-k / N) t} p_{k}+u_{(1-(k+1) / N) t} p_{k+1}-u_{(1-k / N) t}\right) s\left(p_{k}\right)\right\|^{2} \\
& =\left\|\left\{\left(u_{(1-(k-1) / N) t}-u_{(1-k / N) t}\right) p_{k-1}+\left(u_{(1-(k+1) / N) t}-u_{(1-k / N) t}\right) p_{k+1}\right\} s\left(p_{k}\right)\right\|^{2} \\
& \approx \| s\left(p_{k}\right)\left\{p_{k-1}\left(u_{(1-(k-1) / N) t}-u_{(1-k / N) t}\right)^{*}\left(u_{(1-(k-1) / N) t}-u_{(1-k / N) t}\right) p_{k-1}\right. \\
& \left.+p_{k+1}\left(u_{(1-(k+1) / N) t}-u_{(1-k / N) t}\right)^{*}\left(u_{(1-(k+1) / N) t}-u_{(1-k / N) t}\right) p_{k+1}\right\} s\left(p_{k}\right) \| \\
& \leq\left(\frac{C+\epsilon^{\prime}}{N}\right)^{2}
\end{aligned}
$$


where $p_{-1}=0=p_{N+1}$. Since $C / N<\epsilon^{\prime} / 2$, we may assume that

$$
\left\|\left(z_{t}-u_{(1-k / N) t}\right) s\left(p_{k}\right)\right\|<\epsilon^{\prime} .
$$

Since

$$
\begin{aligned}
\left\|z_{t}^{*} z_{t}-1\right\| & \approx\left\|\sum_{k=0}^{N} p_{k}^{1 / 2}\left(z_{t}^{*} z_{t}-1\right) p_{k}^{1 / 2}\right\| \\
& =\left\|\sum_{k=0}^{N} p_{k}^{1 / 2}\left\{\left(z_{t}^{*}-u_{(1-k / N) t}^{*}\right) z_{t}+u_{(1-k / N) t}^{*}\left(z_{t}-u_{(1-k / N) t}\right)\right\} p_{k}^{1 / 2}\right\| \\
& <\epsilon^{\prime}\left(2+\epsilon^{\prime}\right)
\end{aligned}
$$

we may assume that $\left\|z_{t}^{*} z_{t}-1\right\|<3 \epsilon^{\prime}$. For $0 \leq s<t \leq 1$, let

$$
y(s, t)=\sum_{k=0}^{N} p_{k}^{1 / 2}\left(u_{(1-k / N) t}-u_{(1-k / N) s}\right)^{*}\left(u_{(1-k / N) t}-u_{(1-k / N) s}\right) p_{k}^{1 / 2} .
$$

Then we have that

$$
\|y(s, t)\| \leq\left(C+\epsilon^{\prime}\right)^{2}(t-s)^{2}
$$

and

$$
\begin{aligned}
& \left\|\left(z_{t}-z_{s}\right)^{*}\left(z_{t}-z_{s}\right)-y(s, t)\right\| \\
& \approx\left\|\sum p_{k}^{1 / 2}\left(z_{t}-z_{s}\right)^{*}\left(z_{t}-z_{s}\right) p_{k}^{1 / 2}-y(s, t)\right\| \\
& =\| \sum p_{k}^{1 / 2}\left\{\left(\left(z_{t}-z_{s}\right)^{*}-\left(u_{(1-k / N) t}-u_{(1-k / N) s}\right)^{*}\right)\left(z_{t}-z_{s}\right)\right. \\
& \left.\quad+\left(u_{(1-k / N) t}-u_{(1-k / N) s}\right)^{*}\left(z_{t}-z_{s}-\left(u_{(1-k / N) t}-u_{(1-k / N) s}\right)\right)\right\} p_{k}^{1 / 2} \| \\
& \leq 2 \epsilon^{\prime}\left(\left\|z_{t}-z_{s}\right\|+\left(C+\epsilon^{\prime}\right)(t-s)\right) .
\end{aligned}
$$

Hence we have that

$$
\left\|z_{t}-z_{s}\right\|^{2}<\left(C+\epsilon^{\prime}\right)^{2}(t-s)^{2}+2 \epsilon^{\prime}\left(C+\epsilon^{\prime}\right)(t-s)+2 \epsilon^{\prime}\left\|z_{t}-z_{s}\right\|+\epsilon^{\prime \prime},
$$

or

$$
\left(\left\|z_{t}-z_{s}\right\|-\epsilon^{\prime}\right)^{2}<\left(C+\epsilon^{\prime}\right)^{2}(t-s)^{2}+2 \epsilon^{\prime}\left(C+\epsilon^{\prime}\right)(t-s)+\epsilon^{\prime 2}+\epsilon^{\prime \prime}
$$

for an arbitrarily small constant $\epsilon^{\prime \prime}>0$. Assuming that $t-s>\sqrt{2 \epsilon^{\prime}}$ and $\epsilon^{\prime 2}>\epsilon^{\prime \prime}$, we have that

$$
\left(\left\|z_{t}-z_{s}\right\|-\epsilon^{\prime}\right)^{2}<\left(C+\epsilon^{\prime}+\sqrt{\epsilon^{\prime}}\right)^{2}(t-s)^{2}
$$

which implies that

$$
\begin{aligned}
\left\|z_{t}-z_{s}\right\| & <\left(C+\epsilon^{\prime}+\sqrt{\epsilon^{\prime}}\right)(t-s)+\epsilon^{\prime} \\
& <\left(C+\epsilon+2 \sqrt{\epsilon^{\prime}}\right)(t-s) \\
& <\left(C+3 \sqrt{\epsilon^{\prime}}\right)(t-s) .
\end{aligned}
$$


We choose a sequence $\left(t_{i}\right)_{i=0}^{m}$ in $[0,1]$ such that $t_{0}=0<t_{1}<\cdots<t_{m}=1$ and

$$
\sqrt{2 \epsilon^{\prime}}<t_{i}-t_{i-1}<2 \sqrt{\epsilon^{\prime}}<\frac{\epsilon}{C+8 \sqrt{\epsilon^{\prime}}}
$$

Noting that $\left\|\left|z_{t_{i}}\right|-1\right\|<3 \epsilon^{\prime} \ll \epsilon$, define $v_{t_{i}}=z_{t_{i}}\left|z_{t_{i}}\right|^{-1} \in \mathcal{U}\left(B_{0}\right)$. Then we have that for $x \in \mathcal{F}$,

$$
\begin{aligned}
\left\|v_{t_{i}} x-x v_{t_{i}}\right\| & \leq 2\left\|v_{t_{i}}-z_{t_{i}}\right\|+\left\|z_{t_{i}} x-x z_{t_{i}}\right\| \\
& \leq 2\left\|1-\left|z_{t_{i}}\right|\right\|+\left\|\left[z_{t_{i}}, x\right]\right\| \\
& <6 \epsilon^{\prime}+\epsilon<2 \epsilon
\end{aligned}
$$

and that

$$
\begin{aligned}
\left\|v_{t_{i}}-v_{t_{i-1}}\right\| & \leq\left\|v_{t_{i}}\left(1-\left|z_{t_{i}}\right|\right)\right\|+\left\|z_{t_{i}}-z_{t_{i-1}}\right\|+\left\|v_{t_{i-1}}\left(1-\left|z_{t_{i-1}}\right|\right)\right\| \\
& \leq\left(C+3 \sqrt{\epsilon^{\prime}}\right)\left(t_{i}-t_{i-1}\right)+6 \epsilon^{\prime} .
\end{aligned}
$$

Thus, since $5 \sqrt{2}>6$, we have that

$$
\left\|v_{t_{i}}-v_{t_{i-1}}\right\|<\left(C+8 \sqrt{\epsilon^{\prime}}\right)\left(t_{i}-t_{i-1}\right)<\epsilon .
$$

Let

$$
\sqrt{-1} h_{i}=\ln \left(v_{t_{i}} v_{t_{i-1}}^{*}\right)=\ln \left(1-\left(1-v_{t_{i}} v_{t_{i-1}}^{*}\right)\right)
$$

then

$$
\begin{aligned}
\left\|h_{i}\right\| & \leq\left\|1-v_{t_{i}} v_{t_{i-1}}^{*}\right\|+\left\|1-v_{t_{i}} v_{t_{i-1}}^{*}\right\|^{2} \\
& <\left(C+8 \sqrt{\epsilon^{\prime}}\right)\left(t_{i}-t_{i-1}\right)+\left(C+8 \sqrt{\epsilon^{\prime}}\right)^{2} 2 \sqrt{\epsilon^{\prime}}\left(t_{i}-t_{i-1}\right) \\
& <\left(C+\left(3 C^{2}+8\right) \sqrt{\epsilon^{\prime}}\right)\left(t_{i}-t_{i-1}\right) .
\end{aligned}
$$

We define, for $t \in\left[t_{i-1}, t_{i}\right]$,

$$
v_{t}=\exp \left(\sqrt{-1}\left(\frac{t-t_{i-1}}{t_{i}-t_{i-1}}\right) h_{i}\right) v_{t_{i-1}} .
$$

Then $\left(v_{t}\right)$ is a path in $\mathcal{U}\left(B_{0}\right) \cap\left(B_{0}+1\right)$. Moreover $\left(v_{t}\right)$ is a rectifiable path satisfying that

$$
\operatorname{length}\left(\left(v_{y}\right)_{y \in[s, t]}\right)<\left(C+\left(3 C^{2}+8\right) \sqrt{\epsilon^{\prime}}\right)(t-s) \text {. }
$$

If $t \in\left[t_{i-1}, t_{i}\right]$, then $\left\|v_{t}-v_{t_{i-1}}\right\|<\epsilon$ and hence $\left\|\operatorname{Ad} v_{t}(x)-x\right\|<4 \epsilon$ for $x \in \mathcal{F}$. Since $\pi\left(z_{1}\right) \xi_{1}=\eta_{1}$ and $\left\|\left|z_{t}\right|-1\right\|<3 \epsilon^{\prime}$, we have that $\left\|\pi\left(v_{1}\right) \xi_{1}-\eta_{1}\right\|<3 \epsilon^{\prime}$. This is how to construct the path $\left(v_{t}\right)$ in $\mathcal{U}\left(B_{0}\right)$ in the case $\left\langle\pi\left(e_{j-1}\right) \xi, \xi\right\rangle>\epsilon^{\prime 2}$. Otherwise we simply set $v_{t}=1 \in \mathcal{U}\left(B_{0}\right)$.

Let $B_{1}$ be the closure of $\left(1-e_{j+N}\right) B\left(1-e_{j+N}\right)$. If $\left\langle\left(1-\pi\left(e_{j+N}\right)\right) \xi, \xi\right\rangle>\epsilon^{\prime 2}$, set $f=(1-$ $\left.e_{j+N}\right)^{1 / 2} \in B_{1}+1, \xi_{2}=\|\pi(f) \xi\|^{-1} \pi(f) \xi$, and $\eta_{2}=\|\pi(f) \eta\|^{-1} \pi(f) \eta$. There is a rectifiable 
path $\left(w_{t}\right)$ in $\mathcal{U}\left(B_{1}\right)$ such that $w_{1}=1, \pi\left(w_{1}\right) \xi_{2}=\eta_{2}$, and length $\left(\left(w_{u}\right)_{u \in[s, t]}\right) \leq \pi(t-s)$. If $\left\langle\left(1-\pi\left(e_{j+N}\right)\right) \xi, \xi\right\rangle \leq \epsilon^{\prime 2}$, set $w_{t}=1 \in \mathcal{U}\left(B_{1}\right)$. Let $u_{t}=\left(v_{t}-1\right)+\left(w_{t}-1\right)+1 \in \mathcal{U}(B)$.

Note that if $\left\langle\pi\left(e_{j-1}\right) \xi, \xi\right\rangle>\epsilon^{\prime 2}$,

$$
\|\|\left(e_{j-1}^{1 / 2}\right) \xi\|-\| \pi\left(e_{j-1}^{1 / 2}\right) \eta \| \mid<\delta \epsilon^{\prime} / 2<\epsilon^{\prime}
$$

and that if $\left\langle\pi\left(1-e_{j+N}\right) \xi, \xi\right\rangle>\epsilon^{\prime 2}$,

$$
\|\| \pi\left(\left(1-e_{j+N}\right)^{1 / 2}\right) \xi\|-\| \pi\left(\left(1-e_{j+N}\right)^{1 / 2}\right) \eta \| \mid<\delta \epsilon^{\prime} / 2<\epsilon^{\prime} .
$$

Note also that

$$
\begin{aligned}
& \left\|\xi-\pi\left(e_{j-1}^{1 / 2}\right) \xi-\pi\left(\left(1-e_{j+N}\right)^{1 / 2}\right) \xi\right\|^{2} \\
& =1+\left\langle\pi\left(e_{j-1}\right) \xi, \xi\right\rangle+\left\langle\left(1-\pi\left(e_{j+N}\right) \xi, \xi\right\rangle-2\left\langle\pi\left(e_{j-1}^{1 / 2}\right) \xi, \xi\right\rangle-2\left\langle\pi\left(\left(1-e_{j+N}\right)^{1 / 2}\right) \xi, \xi\right\rangle\right. \\
& \leq\left\langle\pi\left(e_{j+N}-e_{j-1}\right) \xi, \xi\right\rangle<\epsilon^{\prime 2} / 2 .
\end{aligned}
$$

Thus $\pi\left(u_{1}\right) \xi \approx \eta$ up to the order $\epsilon^{\prime}$. Since $C \geq \pi$ and $\left\|u_{t}-u_{s}\right\|=\max \left(\left\|v_{t}-v_{s}\right\|,\left\|w_{t}-w_{s}\right\|\right)$, it follows that length $\left(\left(u_{t}\right)\right)<C+\left(3 C^{2}+8\right) \sqrt{\epsilon^{\prime}}$. Since $w_{t}$ commutes with $\mathcal{F}$, it also follows that $\left\|\operatorname{Ad} u_{t}(x)-x\right\|<4 \epsilon, x \in \mathcal{F}$. We then find a rectifiable path $\left(w_{t}\right)$ in $\mathcal{U}(B)$ such that $w_{0}=1, \pi\left(w_{1} u_{1}\right) \xi=\eta,\left\|w_{t}-1\right\|$ is of order $\epsilon^{\prime}$, and length $\left(\left(w_{t}\right)\right)$ is of order $\epsilon^{\prime}$, and then form a new path connecting $\left(u_{t}\right)$ with $\left(w_{t} u_{1}\right)$, which is the desired path. This completes the proof.

Proof of Proposition 8.5 If the hereditary $C^{*}$-subalgebra $A_{1}$ has a unit, this follows from (the proof of) 2.11. If $A_{1}$ has an approximate identity consisting of projections, this also follows from 2.11. Thus we assume at least that $A_{1}$ has no unit.

Let $\mathcal{F}$ be a finite subset of $A_{1}$ and $\epsilon>0$. We may assume that $\|x\| \leq 1$ for $x \in \mathcal{F}, \epsilon>0$ is sufficiently small, and that there is an $e \in A_{1}$ such that $0 \leq e \leq 1$ and $e x e=x, x \in \mathcal{F}$. Let $B$ be a $C^{*}$-algebra containing $A_{1}$. By the previous lemma we may assume that $B=\overline{A_{1} B A_{1}}$.

Let $D$ be a $C^{*}$-algebra such that $B$ is a hereditary $C^{*}$-subalgebra of $D, D \supset A$, and the following diagram is commutative:

$$
\begin{array}{lll}
A_{1} & \subset & B \\
\cap & & \cap \\
A & \subset & D
\end{array}
$$

Let $\epsilon^{\prime}>0$ be such that $2 \sqrt{\epsilon^{\prime}}\left(C+8 \sqrt{\epsilon^{\prime}}\right)<\epsilon$ and let $M, N \in \mathbf{N}$ be so large that $M \epsilon^{\prime 2}>4$ and $N \epsilon^{\prime}>2 C$. There is a sequence $\left(e_{0}, e_{1}, \ldots, e_{M(N+1)}\right)$ in $A_{1}$ such that $e_{0} x e_{0}=x, x \in \mathcal{F}, 0 \leq e_{i} \leq 1$, and $e_{i} e_{i-1}=e_{i-1}$. Let $d$ be a very large constant and let $\mathcal{F}_{1}=\mathcal{F} \cup\left\{d e_{i} \mid 0 \leq i \leq M(N+1)\right\}$. For $\left(\mathcal{F}_{1}, \epsilon\right)$ with $\mathcal{F}_{1}$ as a subset of $A$, we choose $\left(\mathcal{G}_{1}, \delta\right)$ as in Property 8.1 for $(A, C)$. We set

$$
\mathcal{G}=\left\{e_{M(N+1)} x e_{M(N+1)} \mid x \in \mathcal{G}_{1}\right\} \cup\left\{e_{i} \mid 0 \leq i \leq M(N+1)\right\},
$$


which is a finite subset of $A_{1}$.

Let $\pi$ be an irreducible representation of $B$ and $\xi, \eta$ unit vectors in $\mathcal{H}_{\pi}$. We extend $\pi$ to an irreducible representation $\rho$ of $D ; \mathcal{H}_{\pi} \subset \mathcal{H}_{\rho}$. Suppose that

$$
|\langle\pi(x) \xi, \xi\rangle-\langle\pi(x) \eta, \eta\rangle|<\delta \epsilon^{\prime 2} / 2, \quad x \in \mathcal{G} .
$$

There exists a $j \in\{1,2, \ldots,(M-1)(N+1)\}$ such that

$$
\left\langle\pi\left(e_{j+N}-e_{j-1}\right) \xi, \xi\right\rangle+\left\langle\pi\left(e_{j+N}-e_{j-1}\right) \eta, \eta\right\rangle<\epsilon^{\prime 2} / 2 \text {. }
$$

If $\left\langle\pi\left(e_{j-1}\right) \xi, \xi\right\rangle>\epsilon^{\prime 2}$, then we have that for the normalized vectors $\xi_{1}, \eta_{1}$ for $\pi\left(e_{j-1}^{1 / 2}\right) \xi$, $\pi\left(e_{j-1}^{1 / 2}\right) \eta$ respectively,

$$
\left|\left\langle\pi(x) \xi_{1}, \xi_{1}\right\rangle-\left\langle\pi(x) \eta_{1}, \eta_{1}\right\rangle\right|<\delta, \quad x \in \mathcal{G},
$$

which implies that

$$
\left|\left\langle\rho(x) \xi_{1}, \xi_{1}\right\rangle-\left\langle\rho(x) \eta_{1}, \eta_{1}\right\rangle\right|<\delta, \quad x \in \mathcal{G}_{1} .
$$

Then there is a rectifiable path $\left(u_{t}\right)$ in $\mathcal{U}(D)$ such that $u_{0}=1, \rho\left(u_{1}\right) \xi_{1}=\eta_{1}$, $\| \operatorname{Ad} u_{t}(x)-$ $x \|<\epsilon, x \in \mathcal{F}_{1}$, and length $\left(\left(u_{y}\right)_{y \in[s, t]}\right)<\left(C+\epsilon^{\prime}\right)(t-s)$ for $0 \leq s<t \leq 1$. Let $B_{0}$ be the closure of $e_{j+N-1} D e_{j+N-1}$, which is a hereditary $C^{*}$-subalgebra of $B$. Then, as in the proof of the previous lemma, we obtain a rectifiable path $\left(v_{t}\right)$ in $\mathcal{U}\left(B_{0}\right)$ such that $v_{0}=1, \pi\left(v_{1}\right) \xi_{1}=\eta_{1}, v_{t}-1 \in B_{0},\left\|\operatorname{Ad} v_{t}(x)-x\right\|<4 \epsilon, x \in \mathcal{F}$, and length $\left(\left(v_{y}\right)_{y \in[s, t]}\right)<(C+$ $\left.\left(3 C^{2}+8\right) \sqrt{\epsilon^{\prime}}\right)(t-s)$ (by making $d$ sufficiently large). On the other hand if $\left\langle\pi\left(e_{j-1}\right) \xi, \xi\right\rangle \leq$ $\epsilon^{\prime 2}$, we set $v_{t}=1 \in \mathcal{U}\left(B_{0}\right)$.

Let $B_{1}$ be the closure of $\left(1-e_{j+N}\right) B\left(1-e_{j+N}\right)$, which is a hereditary $C^{*}$-subalgebra of $B$. If $\left\langle\left(1-\pi\left(e_{j+N}\right)\right) \xi, \xi\right\rangle>\epsilon^{\prime 2}$, let $\xi_{2}$ and $\eta_{2}$ be the normalized vectors for $\pi\left(\left(1-e_{j+N}\right)^{1 / 2}\right) \xi$ and $\pi\left(\left(1-e_{j+N}\right)^{1 / 2}\right) \eta$ respectively. There is a rectifiable path $\left(w_{t}\right)$ in $\mathcal{U}\left(B_{1}\right)$ such that $w_{0}=1, \pi\left(w_{1}\right) \xi_{2}=\eta_{2}, w_{t}-1 \in B_{1}$, and length $\left(\left(w_{y}\right)_{y \in[s, t]}\right) \leq \pi(t-s)$. Note that $\left(w_{t}-1\right) x=0=x\left(w_{t}-1\right), x \in \mathcal{F}$. If $\left\langle\left(1-\pi\left(e_{j+N}\right)\right) \xi, \xi\right\rangle \leq \epsilon^{\prime 2}$, we set $w_{t}=1 \in \mathcal{U}\left(B_{1}\right)$. Let $u_{t}=\left(v_{t}-1\right)+\left(w_{t}-1\right)+1 \in \mathcal{U}(B)$, from which one can construct the desired path as in the proof of the previous lemma.

Hence Propositions 2.11 and 3.5 can be extended as follows:

Remark 8.7 If $\mathcal{C}_{C}$ denotes the class of $C^{*}$-algebras satisfying Property 8.1 for a constant $C \geq \pi$, then the following statements hold:

1. If $A$ is a non-unital $C^{*}$-algebra, $A \in \mathcal{C}_{C}$ if and only if $\tilde{A} \in \mathcal{C}_{C}$, where $\tilde{A}$ is the $C^{*}$-algebra obtained by adjoining a unit to $A$.

2. If $A_{1}, A_{2} \in \mathcal{C}_{C}$, then $A_{1} \oplus A_{2} \in \mathcal{C}_{C}$.

3. If $A \in \mathcal{C}_{C}$ and $A_{1}$ is a hereditary $C^{*}$-subalgebra of $A$, then $A_{1} \in \mathcal{C}_{C}$.

4. If $A \in \mathcal{C}_{C}$ and $I$ is an ideal of $A$, then $I, A / I \in \mathcal{C}_{C}$. 
5. If $\left(A_{n}\right)$ is an inductive system with $A_{n} \in \mathcal{C}_{C}$, then $\lim _{n} A_{n} \in \mathcal{C}_{C}$.

Remark 8.8 If $A$ is a (unital or non-unital) $C^{*}$-algebra with Property 8.1 and $C$ is a (unital or non-unital) commutative $C^{*}$-algebra, then $A \otimes C$ has Property 8.1 for the same constant.

\section{References}

[1] O. Bratteli, Inductive limits of finite-dimensional $C^{*}$-algebras, Trans. Amer. Math. Soc. 171 (1972), 195-234.

[2] O. Bratteli, G.A. Elliott, D.E. Evans, and A. Kishimoto, On the classification of $C^{*}$-algebras of real rank zero, III: The infinite case, Fields Inst. Commun. 20 (1998), 11-72.

[3] O. Bratteli and A. Kishimoto, Homogeneity of the pure state space of the Cuntz algebra, J. Funct. Anal. 171 (2000), 331-345.

[4] O. Bratteli, A. Kishimoto, and D.W. Robinson, Abundance of invariant and almost invariant pure states of $C^{*}$-dynamical systems, Commun. Math. Phys. 187 (1997), 491-507.

[5] M. Dadarlat and G. Gong, A classification result for approximately homogeneous $C^{*}$ algebras of real rank zero, Geom. Funct. Anal. 7 (1997), 646-711.

[6] G.A. Elliott, On the classification of $C^{*}$-algebras of real rank zero, J. reine angew. Math. 443 (1993), 179-219.

[7] D.E. Evans and A. Kishimoto, Trace-scaling automorphisms of certain stable AF algebras, Hokkaido Math. J. 26 (1997), 211-224.

[8] E. Kirchberg and N.C. Phillips, Embedding of exact $C^{*}$-algebras in the Cuntz algebra $\mathcal{O}_{2}$, J. reine angew. Math. 525 (2000), 17-53.

[9] A. Kishimoto and A. Kumjian, The Ext class of approximately inner automorphisms, II, to appear in J. Operator Theory.

[10] N.C. Phillips, A classification theorem for nuclear purely infinite simple $C^{*}$-algebras, Documenta Mathematica 5 (2000), 49-114 (electronic).

[11] R.T. Powers, Representation of uniformly hyperfinite algebras and their associated von Neumann rings, Ann. of Math. 86 (1967), 138-171.

[12] M. Rørdam, Classification of certain infinite simple $C^{*}$-algebras, III, Fields Inst. Commun. 13 (1997) 257-282.

[13] S. Sakai, $C^{*}$-algebras and $W^{*}$-algebras, Classics in Math., Springer, 1998. 